\title{
Experimental research of assembled buckling-restrained braces wrapped with carbon or basalt fiber
}

\author{
Mingming Jia*, Xiaohui Yu, Dagang Lu, Binbin Lu
}

\begin{abstract}
A new assembled buckling-restrained brace (ABRB) wrapped with carbon or basalt fiber cloth is proposed. It has the following advantages: 1) it can be disassembled by cutting the fiber cloth; 2) it is easy to inspect and repair the core plates after destructive earthquakes; 3 ) the outer fiber material also serves as a corrosion resistance to the outer steel tube. A total of eight ABRB specimens were tested under quasi-static axial loads. The test results indicate that the ABRB is capable to undergo fully-reverse yielding cycles without loss of stiffness and strength with sufficient ductility and energy absorption capacity. Multi-wave buckling occurred on the core plates of ABRBs resulting in "jumped" hysteretic curves in compression stage. ABRB specimens fractured in the middle of the core plate elements when a loading protocol included increasing deformation amplitudes with additional fatigue cyclic loading; while when the loading protocol consisted of increasing deformation amplitude cyclic loading without maximum amplitude limit, rupture of the ABRB specimens occurred at the junctions of yielding segments and non-yielding segments of core plate. When a pair of restraining members of an ABRB are connected and restrained with fiber cloth, the assembled constraint mechanics can resist the lateral thrust exerted by core plate. The nonlinear finite element analysis results show good agreement with the experimental data. Both the results from the experiment and numerical simulation show that the proposed ABRBs can satisfy the performance requirements for the BRB components and provide a beneficial alternative for BRB fabrication and application.
\end{abstract}

Keywords: Assembled buckling restrained brace (ABRB); carbon fiber; basalt fiber; hysteretic behavior; multi-wave buckling 


\section{Introduction}

Buckling restrained braces (BRBs) are made from encasing a core steel cross-shape or flat bar member into a tube and confined by infill concrete. The steel core member, whose tension and compression yield capacity are almost identical with each other, is designed to resist the axial forces. An unbounding material or clearance is commonly assigned between the core member and the infill concrete with the aim of reducing the bonding-induced friction. The infill concrete together with the outer steel tube encasement provide sufficient flexural strength and stiffness to prevent global buckling of a brace, and have a positive effect on the resistance of local buckling. Previous research and engineering applications have shown that BRBs can improve the seismic performance of conventional buildings [1-3].

Numerous experimental and numerical studies have been conducted to examine the mechanical property, stability performance and hysteretic behavior of different types of BRBs. For example, Watanabe et al. [4] addressed the effect of outer tube configuration on overall load capacity of BRBs. Chen et al. [5] tested the hysteretic behavior of the BRBs made of low yield point (LYP) steel. Through a comprehensive test involving 20 specimens, Wang et al. [6] presented a reliable constitutive model of LYP steel applied in the simulation of BRBs. Lai et al. [7] proposed a new type of BRB employing double steel cores encased in twin steel tubes and infill concrete. Black et al. [8] reported the results from a comprehensive component testing program involving five BRBs with various configurations and verified the reliability and practicability of the BRBs (known as 'Unbonded Braces'). Jia et al. $[9,10]$ experimentally investigated the cyclic behavior of the BRBs with $\mathrm{H}$ cross section unrestrained segments and compared them with that with cruciform cross section unrestrained segments. Genna and Gelfi [11] conducted experimental and numerical studies on the lateral forces exerted by the core plate of BRBs. To investigate core plate multi-mode buckling phenomenon, Wu et al. [12] conducted cyclic loading tests and finite element analyses on six all-steel BRBs under different loading patterns. The good strength and stiffness, hysteric characteristics and energy dissipation capacity shown in the above studies have accelerated the application of BRBs in civil engineering [1].

Recently, there has been an increasing focus on the concept of assembled BRBs (shortly named as ABRBs hereafter). Unlike the conventional integral BRBs, the ABRBs can be repaired or replaced conveniently by disassembling outer restraining members after core plates being damaged in earthquakes. Besides, the subassemblies of ABRBs can be fabricated and delivered separately for on-site erection and 
are thus valuable for quality control in practical use. Several studies have been conducted on the development of different types of ABRBs. For example, Iwata and Murai [13] developed an ABRB by sandwiching a core plate with a pair of welding mortar-filled channel steel as a restraining part. Chou and Chen [14] presented the experimental and numerical results on the performance of the BRBs composed of a steel core plate and two identical restraining members which sandwich the core plate with fully tensioned high-strength bolts to prevent core buckling. The subassemblies of ABRBs are commonly connected with welded or bolted attachments. Eryasa and Topkaya [15] conducted a seismic test program performed on 12 steel-encased BRBs for examining the effects of bolt pretension, core-to-encasing attachment details, aspect ratio of core segment and imperfections due to manufacturing on the hysteric performance of BRBs. Hoveidae and Rafezy [16] presented finite element analysis results of the hysteric behaviors of one kind of full-steel ABRBs considering different buckling restraining mechanisms. Among the available ABRBs, the aluminum alloy has also been used since it is lighter than the normal steel. A kind of high-performance BRBs with stoppers that are bolt-assembled was proposed by Usami et al. [17], and the effect of stoppers on the low-cycle fatigue performance were experimentally and numerically examined [18]. Wang et al. [19] proposed an extruded aluminum alloy core plate ABRB, which can avoid the use of welding and complex BRB ends in previous BRB research. In the study of Dusicka et al. [20, 21], an aluminum alloy core was adopted for a new type of ABRB constrained with the glass fiber reinforced polymer pultruded tubes used as the assembled buckling-restraint mechanics. Deng and Pan [22] proposed GFRP steel BRB restrained with four GFRP pultruded tubes, which are tied together by GFRP wrapping layers, are used to restrict core steel component buckling instead of conventional steel tube and infilled concrete or mortar. Although the afore-mentioned studies have been conducted, there is still a long way to apply the ABRBs in practical engineering since the configurations and fabrication are commonly complicated. Moreover, the outer restraining members of the welding-ABRBs will be destroyed when replacing the core plate element. For the bolting-ABRBs, the fabrication precision and cost is generally high and thus unattractive for civil engineers.

This study aims to propose a new ABRB without using traditional welding or bolting method for assembling subassemblies. As shown in Fig. 1, the new ABRB is composed of three components, i.e., the core plate element, a pair of concrete infilled channel steel, and the wrapped carbon or basalt fiber cloth. The pair of concrete infilled channel steel sandwiches the core plate element and serves as a restraining member. The wrapped carbon or basalt fiber cloth provides the constraints for subassembly connection. 
Since the carbon or basalt fiber is a cheap and high-strength material, the fiber cloth can significantly reduce the fabrication cost of the ABRB. Meanwhile, the fiber cloth can provide sufficient constraints to prevent the separation of a pair of restraining members and resist the lateral thrust at restraining members exerted by the core plate after its buckling. Compared to the ABRBs assembled by welding or bolts, the presented ABRB is easier to be disassembled by cutting the wrapped fiber cloth. Moreover, the fiber cloth can greatly improve the corrosion resistance of outer steel tube. This is valuable to ensure the durability of the ABRBs even when they are subjected to a hostile environment. A total of eight specimens are designed and tested to examine the mechanical property, hysteretic behavior and energy absorption capacity of the presented ABRB with considering different loading protocols and different constraint ratios. Besides, nonlinear finite element analyses are also conducted for the specimens.

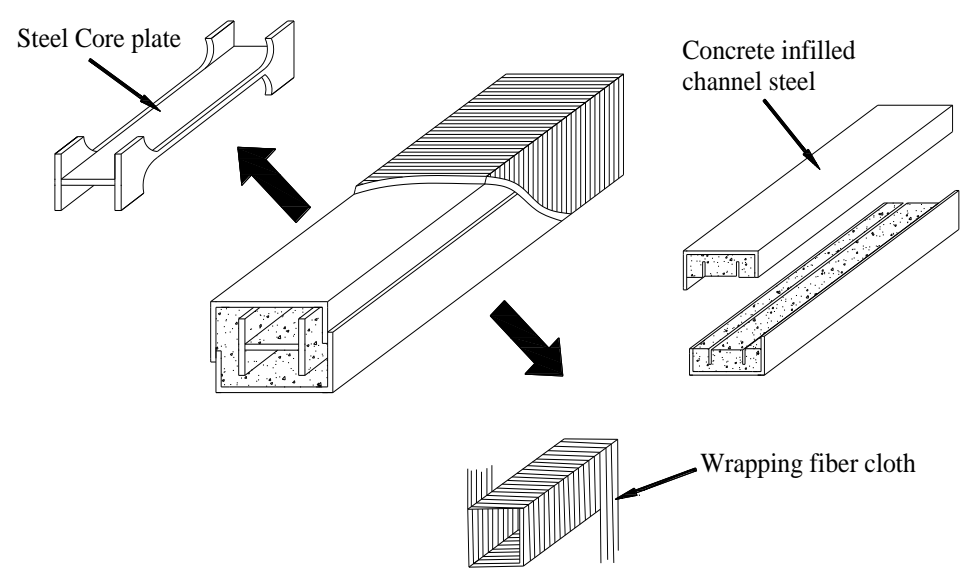

Fig.1. Proposed assembled buckling restrained brace

\section{Design requirements of ABRBs}

\subsection{Global flexural buckling}

The global flexural buckling load $P_{e}$ of BRB specimen is calculated by the following expression:

$$
P_{e}=\frac{\pi\left(0.05 E_{1} I_{1}+E_{r} I_{r}\right)}{L_{t}^{2}}
$$

where $E_{\mathrm{r}} I_{\mathrm{r}}$ denotes the moment resistant stiffness of the restraining members including a pair of concrete infilled channel steel; $E_{1} I_{1}$ denotes the moment resistant stiffness of core plate. The factor of 0.05 is used to consider the decrease of elastic stiffness of core plate during the plastic stage; $L_{t}$ is the total length of BRB specimen.

According to the current Chinese code, 'Chinese technical specification for steel structure of tall 
buildings (JGJ99-2015)' [23], $P_{e}$ should satisfy the following criterion:

$$
\zeta=\frac{P_{e}}{P_{u}} \geq 1
$$

where $\zeta$ is commonly called constraining ratio; and $P_{u}$ is the ultimate load of core plate, which can be calculated by the following equations:

$$
P_{u}=\beta \omega P_{y}
$$

$$
P_{y}=\eta_{y} f_{a y} A_{c}
$$

where $P_{y}$ denotes the yielding load of core plate; $\beta$ is compression adjustment factor; $\omega$ is material strain hardening factor; $\eta_{y}$ is material over-strength factor; $f_{a y}$ is the yield strength of core plate; and $A_{c}$ is cross section area of core plate. According to FEMA450 [24], the values of $\beta$ is considered as $\beta \leq 1.3$, while the values of $\omega$ are taken as $1.2 \leq \omega \leq 1.5$ based on the available experimental data $[1,4-8]$. When $\beta=1.3$ and $\omega=1.5, \beta \omega$ will achieve the maximum value as 1.95 . Submitting $\beta \omega=1.95$ into Eq. (2), the acceptable criterion with respect to $\zeta$ can be conservatively defined by

$$
\zeta=\frac{P_{e}}{P_{y}} \geq 1.95
$$

\subsection{Flexural strength of restraining members}

Let $M$ denote the design moment of restraining members. It should be less than the corresponding flexural strength $M_{u}$, yielding

$$
M \leq M_{u}
$$

where the value of $M$ can be calculated by the following equation

$$
M=\frac{P_{u} P_{e} a}{P_{e}-P_{u}}
$$

where $a$ is original maximum deformation at midpoint of restraining member.

A pair of channel steel sections can form a section of rectangle steel tube. The $M_{u}$ value of the restraining member, i.e., concrete infilled rectangle steel tube, can be calculated by the following equation $[25]$

$$
\begin{gathered}
M_{u}=\left[0.5 A_{s}\left(h-2 t_{s}-d_{n}\right)+w_{r} t_{s}\left(t_{s}+d_{n}\right)\right] f_{s} \\
d_{n}=\frac{A_{s}-2 b_{r} t_{s}}{\left(b_{r}-2 t_{s}\right) \frac{f_{c}}{f_{s}}+4 t_{s}}
\end{gathered}
$$


rectangle steel tube, respectively; $t_{s}$ is thickness of rectangle steel tube; $d_{n}$ is depth of compression zone of concrete; $f_{s}$ is the design value of steel yielding strength $\left(f_{s}\right.$ is $215 \mathrm{~N} / \mathrm{mm}^{2}$ for Q235 steel in [26]); and $f_{c}$ is the design value of concrete compressive strength $\left(f_{c}\right.$ is $14.3 \mathrm{~N} / \mathrm{mm}^{2}$ for $\mathrm{C} 30$ concrete in [27]).

\subsection{Forces acting on restraining members against multi-mode buckling}

Fig. 2(a) shows the multi-mode buckling probably occurred in the core plate when the clearances between the core plate surface and the restraining member, are subjected to a large compressive strain. The lateral thrust is exerted by the core plate on the restraining member, which is perpendicular to core plate and caused by the interface pressure between the core plate and the infill concrete. The interface pressure is assumed to be distributed in a triangle form as shown in Fig. 2(b). This assumption is derived based on a large-scale numerical simulation with consideration of diverse core plate sizes and concrete strengths [23].

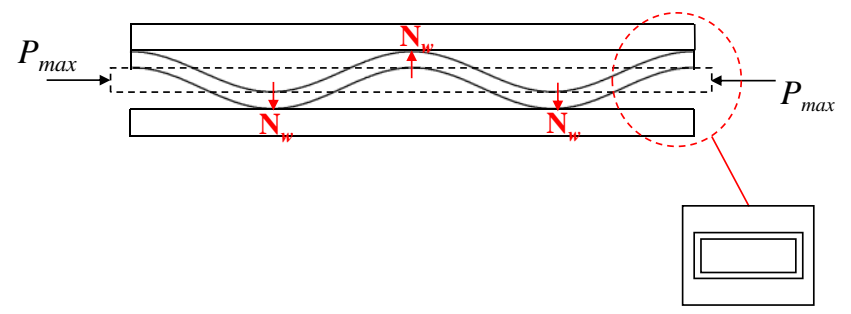

(a) Multi-mode buckling of core plate under compression

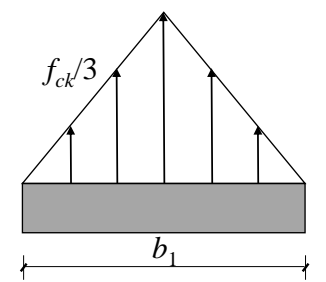

(b) The interface pressure between the core plate and concrete

Fig.2. Forces acting on restraining members against multi-mode buckling

To avoid concrete cracking, the thrust force of core plate is required to be equal with the hoop tensile strength of the fiber cloth. According to the graphical expression in Fig.1b, the lateral thrust, $T$, can be calculated by

$$
T=\frac{1}{2} \times \frac{f_{c k}}{3} \times b_{1}=\frac{f_{c k} b_{1}}{6}
$$

Because the lateral thrust is restrained by two sides of fiber cloth, the thickness of fiber cloth, $t_{f}$, can be calculated by

$$
t_{f}=\frac{1}{2} \times \frac{T}{f_{e}}=\frac{f_{c k} b_{1}}{12 f_{e}}
$$


where $f_{c k}$ is the characteristic value of axial compressive strength of concrete $\left(f_{c k}\right.$ is $20.1 \mathrm{~N} / \mathrm{mm}^{2}$ for C30 concrete [27]); $b_{1}$ is width of core plate; and $f_{e}$ is elastic tensile strength of fiber cloth.

\section{Test Program}

\subsection{Specimen}

The test program consisted of cyclic tests of eight ABRB specimens. Fig. 3 shows the configurations of specimens, where $L_{t}$ is the total length of BRB specimens; $L_{\mathrm{s}}$ and $L_{r y}$ are the lengths of channel steel and restrained yielding segment, respectively; $L_{r n}$ and $L_{u n}$ are the lengths of restrained and unrestrained non-yielding segment of core plate, respectively; $w_{c}$ is width of rectangular cross section of core plate; $t_{c}$ is thickness of rectangular cross section of core plate; $t_{s}$ is thickness of channel steel; $w_{H}$ and $t_{H}$ are the width and thickness of flange of $\mathrm{H}$-shaped section of non-yielding segment; and $a, h$, and $b$ are flange widths of three sides of channel steel. Table 1 summarizes member sizes for each specimen. For convenience, the specimens are shortly labelled as "S (or L) - Bas (or CFRP) - 1(or 2)", where 'S' and 'L' refer the specimens which have shorter and longer core plates with $l_{t}=1200 \mathrm{~mm}$ and $l_{t}=1500 \mathrm{~mm}$, respectively; 'Bas' and 'CFRP' refer to the specimens that are wrapped by basalt and carbon fiber, respectively; and ' 1 ' and ' 2 ' refer to the specimen tested under different loading protocols. The details of the used loading protocols in the test program will be introduced in section 3.2.

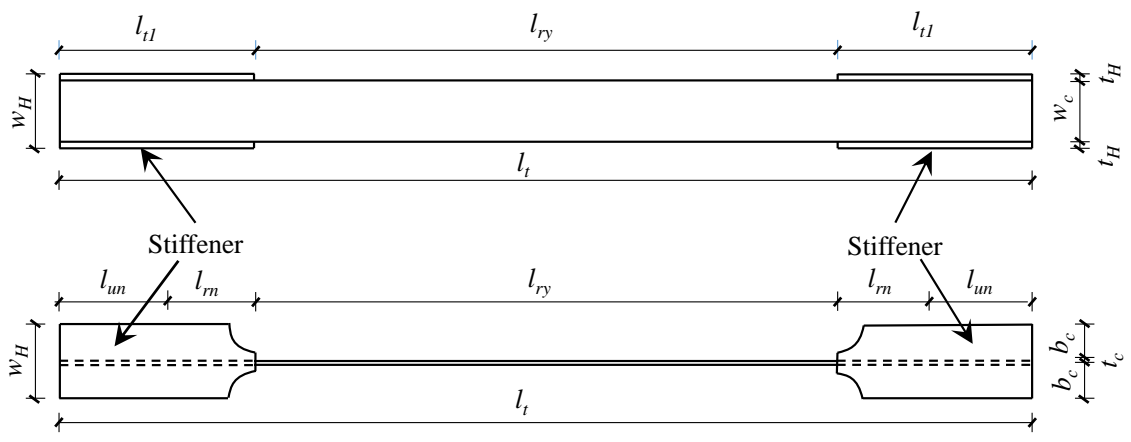

(a) Core plate 


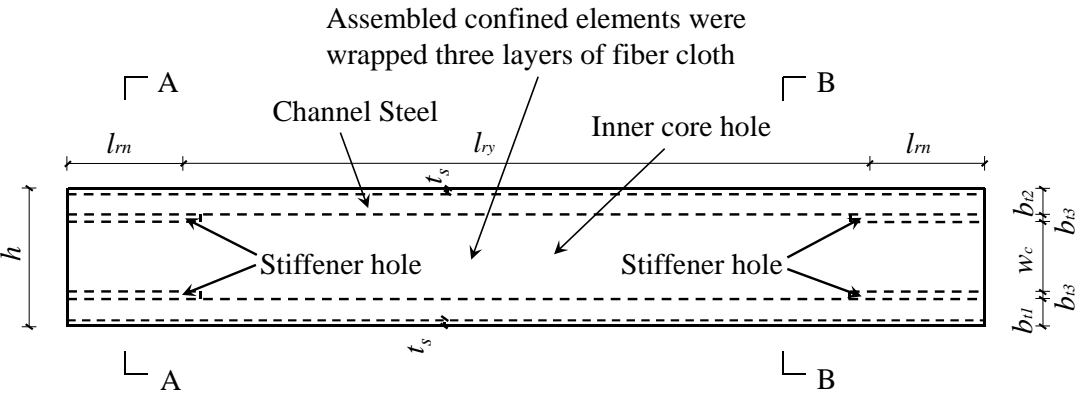

(b) Plan view of restraining members

Assembled confined elements were wrapped three layers of fiber cloth

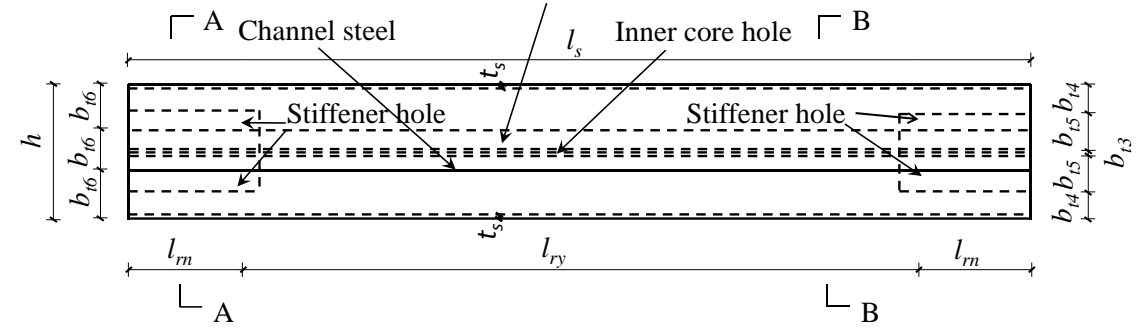

(c) Elevation view of restraining members

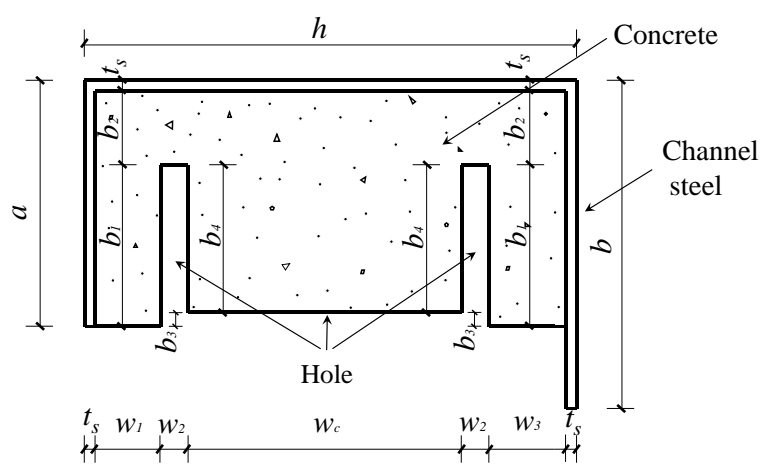

(d) Section A-A

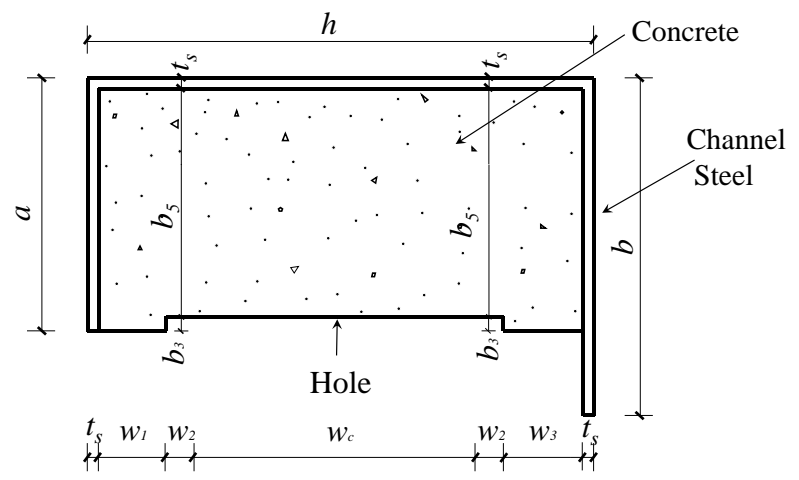

(e) Section B-B

Fig.3. Dimensions of specimens 
Table 1 Specimen sizes $(\mathrm{mm})$

\begin{tabular}{cccccccccc}
\hline Specimens & $w_{H} \times t_{H}$ & $w_{c} \times t_{c}$ & $a \times h \times b \times t_{s}$ & $l_{t}$ & $l_{r y}$ & $l_{r n}$ & $l_{u n}$ & $l_{s}$ & $l_{t 1}$ \\
\hline S-Bas/CFRP-1/2 & $96 \times 8$ & $80 \times 8$ & $90 \times 180 \times 120 \times 4$ & 1200 & 720 & 120 & 120 & 960 & 240 \\
L- Bas/CFRP-1/2 & $116 \times 8$ & $100 \times 8$ & $90 \times 180 \times 120 \times 4$ & 1500 & 900 & 150 & 150 & 1200 & 300 \\
\hline
\end{tabular}

194

195

196

197

Table 1 Specimen sizes (Continued, $\mathrm{mm}$ )

\begin{tabular}{cccccccccccccccc}
\hline Specimens & $b_{c}$ & $b_{t 1}$ & $b_{t 2}$ & $b_{t 3}$ & $b_{t 4}$ & $b_{t 5}$ & $b_{t 6}$ & $w_{1}$ & $w_{2}$ & $w_{3}$ & $b_{1}$ & $b_{2}$ & $b_{3}$ & $b_{4}$ & $b_{5}$ \\
\hline S-Bas/CFRP-1/2 & 44 & 42 & 38 & 10 & 41 & 44 & 60 & 34 & 10 & 38 & 49 & 37 & 5 & 44 & 81 \\
L- Bas/CFRP-1/2 & 54 & 32 & 28 & 10 & 31 & 54 & 60 & 24 & 10 & 28 & 59 & 27 & 5 & 54 & 81 \\
\hline
\end{tabular}

\section{Carbon fiber and basalt fiber were both used in this test program, whose characteristics were obtained} from tensile test and shown in Table 2, where $t_{f}$ is the fiber thickness; $f_{u f}$ is the ultimate elastic strength; $E_{f}$ is the elastic modulus; and $\varepsilon_{u f}$ is the ultimate strain percentage. The steel of Grade Q235B [23] with a nominal yielding strength $f_{y}$ of $235 \mathrm{~N} / \mathrm{mm}^{2}$ was used for fabricating the ABRB specimens. The experimental stress-strain curves for the steel core plate and the outer channel-section steel are shown in Fig. 4(a) and Fig. 4(b), respectively. The measured tensile characteristics of the steel core plate are given in Table 3 , where $f_{y}$ and $f_{u}$ are the yielding strength and ultimate strength, respectively; and $E_{s}$ is the elastic modulus. The target average compressive strength, $f_{c}$, of the adopted infill concrete at age 28 days is $30 \mathrm{MPa}$. Based on the obtained yielding strength $f_{y}$ from the material tensile coupon test (see Table 3), the yielding loads $P_{y}$ of core plate are calculated by multiplying the cross-sectional area $A_{y}\left(=w_{c} \times t_{c}\right)$ and the yield strength $f_{y}$. Table 4 summarizes the specimen strengths, including $P_{y}, P_{u}, P_{e}, M_{u}$ and $M$, where the ultimate loads of core plate $P_{u}$ are computed according to Eq. (3) with $\beta \omega=1.95$, and the global bulking loads of specimen $P_{e}$ are predicted by Eq. (1). It is clear that the predicted values of $P_{e}$ are totally far beyond the corresponding values of $P_{y}$. The ratios of $P_{e} / P_{y}$ are greater than 50 or 30 for the specimens with longer or shorter lengths, respectively. The large values of $P_{e} / P_{y}$ considered for specimens can avoid the occurrence of global bulking during loading process. The design moments $M_{u}$ and the flexural strengths $M$ of the restraining members are computed according to Eq. (7) and Eq. (8), respectively. Table 4 shows the computed values of $M_{u}$ and $M$, where the $M$ values for all specimens are totally greater than the corresponding design values $M_{u}$. This result implies that the flexural strength of restraining members can satisfy the requirement of Eq. (6). According to Eq. (11), the required thickness of fiber cloth is calculated as between $0.031 \mathrm{~mm}$ and $0.041 \mathrm{~mm}$. Since the thickness of one layer of basalt fiber and carbon fiber that are used here are $0.168 \mathrm{~mm}$ and 


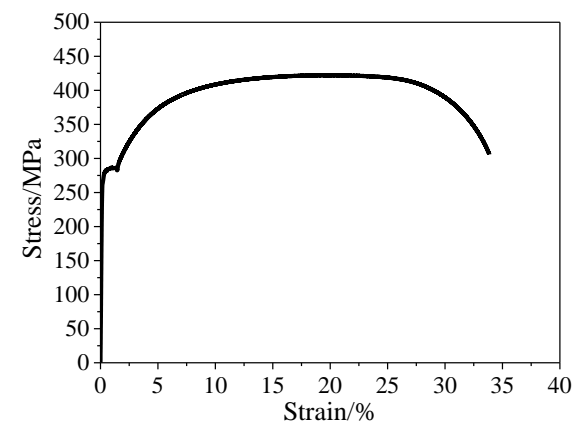

(a) Steel core plate

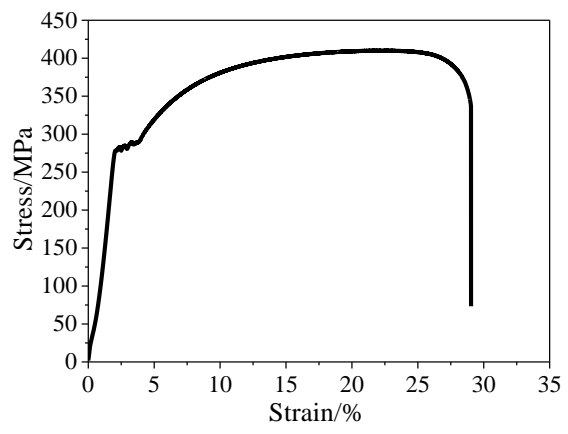

(b) Channel steel

Fig.4. Stress-strain curve of steel

Table 2 Characteristics of basalt and carbon fiber

\begin{tabular}{ccccc}
\hline Fiber & $t_{f}(\mathrm{~mm})$ & $f_{u f}\left(\mathrm{~N} / \mathrm{mm}^{2}\right)$ & $E_{f}\left(\mathrm{~N} / \mathrm{mm}^{2}\right)$ & $\varepsilon_{u f}(\%)$ \\
\hline Basalt fiber & 0.168 & 4340 & $2.44 \times 10^{5}$ & 1.71 \\
Carbon fiber & 0.160 & 4100 & $1.15 \times 10^{5}$ & 2.4 \\
\hline
\end{tabular}

Table 3 Characteristics of steel

\begin{tabular}{cccc}
\hline steel & $f_{y}\left(\mathrm{~N} / \mathrm{mm}^{2}\right)$ & $f_{u}\left(\mathrm{~N} / \mathrm{mm}^{2}\right)$ & $E_{s}\left(\mathrm{~N} / \mathrm{mm}^{2}\right)$ \\
\hline core plate & 280 & 421 & $2.03 \times 10^{5}$ \\
outer channel-section & 284 & 409 & $2.01 \times 10^{5}$ \\
\hline
\end{tabular}

Table 4 Specimen strengths

\begin{tabular}{ccccccccc}
\hline Specimens & S-Bas-1 & S-Bas-2 & S-CFRP-1 & S-CFRP-2 & L-Bas-1 & L-Bas-2 & L-CFRP-1 & L-CFRP-2 \\
\hline$P_{y}(\mathrm{kN})$ & 179.2 & 179.2 & 179.2 & 179.2 & 224 & 224 & 224 & 224 \\
$P_{u}(\mathrm{kN})$ & 340.48 & 340.48 & 340.48 & 340.48 & 425.6 & 425.6 & 425.6 & 425.6 \\
$P_{e}(\mathrm{kN})$ & 9562.112 & 9562.112 & 9562.112 & 9562.112 & 8473.92 & 8473.92 & 8473.92 & 8473.92 \\
$P_{e} / P_{y}$ & 53.6 & 53.6 & 53.6 & 53.6 & 37.83 & 37.83 & 37.83 & 37.83 \\
$a(\mathrm{~mm})$ & 0.93 & 0.91 & 0.66 & 0.92 & 1.11 & 1.06 & 1.05 & 1.10 \\
$M_{u}(\mathrm{kN} \cdot \mathrm{mm})$ & 45269 & 45269 & 45269 & 45269 & 45269 & 45269 & 45269 & 45269 \\
$M(\mathrm{kN} \cdot \mathrm{mm})$ & 339.2 & 321.3 & 240.7 & 324.8 & 497.4 & 475.0 & 470.5 & 492.9 \\
$t_{f}(\mathrm{~mm})$ & 0.031 & 0.031 & 0.033 & 0.033 & 0.039 & 0.039 & 0.041 & 0.041 \\
$t(\mathrm{~mm})$ & 0.504 & 0.504 & 0.48 & 0.48 & 0.504 & 0.504 & 0.48 & 0.48 \\
\hline
\end{tabular}


Fig. 5 shows the fabrication of the core plate used for specimens. The core plate is composed of a centre steel plate and two end strengthened portions. As shown in Fig. 5(a) and (b), two end portions were enhanced by welding two steel plates on the centre steel plate to form an $\mathrm{H}$ cross section. Since a small cross-section was used, the centre part of the core plate would yield firstly and dissipate energy through its plastic deformation. The end portions were enlarged and stiffened with four $8 \mathrm{~mm}$ thick steel plates to supply extra flexural rigidity for buckling strength (see Fig. 5(b)). The core plate was welded onto 20mm thick steel end plate for connection by the loading equipment. Triangle reinforced plates were welded on the end of core plate element and end plate for strengthening (see Fig. 5(c), (d)).

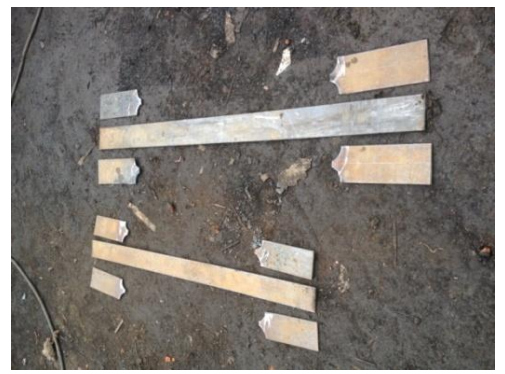

(a) Core plate before welding

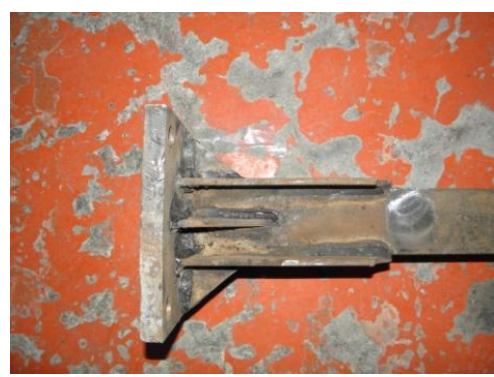

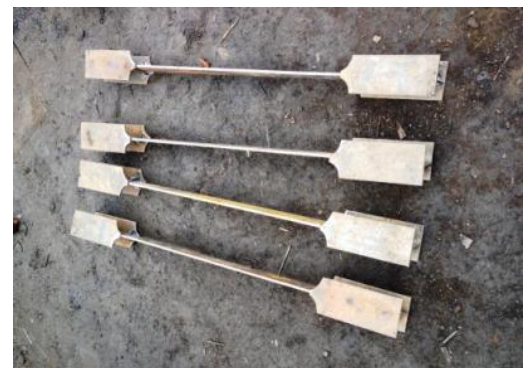

(b) Core plate after welding

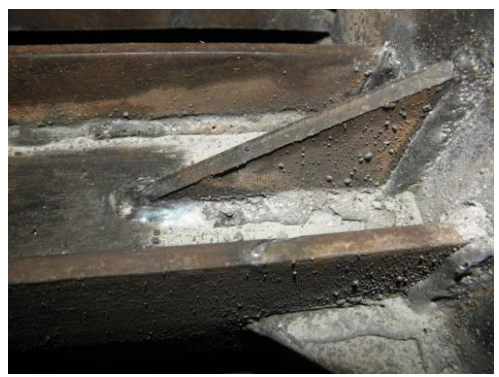

(c) Load-carrying element was welded on the end plate $\quad$ (d) Triangle reinforced plates were welded for strengthening

Fig.5. Fabrication of core plate

Fig. 6 shows the fabrication of the outer channel steel used for specimen. Plain steel plates were first cold bended into U shaped channel-section (see Fig. 6(a)). Two seal plates were then welded onto both ends of channel steel components (see Fig. 6(b)), and form a half restraining member after concrete casting into it. 


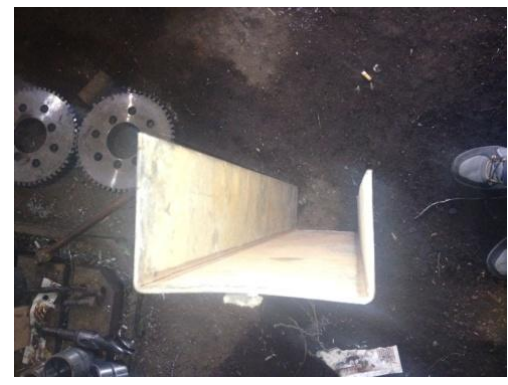

(a) Cross section of channel steel

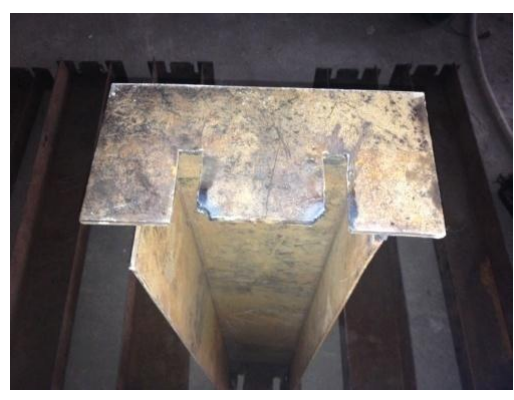

(b) Seal plates were welded onto channel steel

Fig.6. Fabrication of channel steel

When filling concrete into the channel steel, wood plates of $6 \mathrm{~mm}$ thick were pre-located in the concrete for reserving a space of half thickness of core plate. The clearances between the core plate and the restraining member have been reserved to absorb the transverse deformation of core plate and avoid the improvement of compression bearing capacity. After 28 days concrete curing, the wood plates were removed. Fig. 7 shows the assembling process of test specimens. PVC plastic film was used to cover the surface of the core plate and the concrete infilled channel steel to allow a smooth movement between the load-carrying element and the lateral-support element (see Fig. 7(a) and Fig. 7(b)). After the core plate is sandwiched by a pair of channel steel, the subassemblies are connected by spot welding on the overlapping channel steel (see Fig. 7(c)). The welding process should keep the core plate element match the restraining members precisely. Finally, the fabrication of ABRB specimens were finished by wrapping fiber clothes around the confined channel steel for providing constraints.

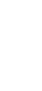




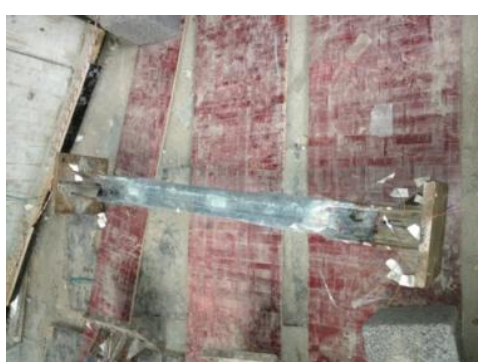

(a) Unbounding process of core plate elements

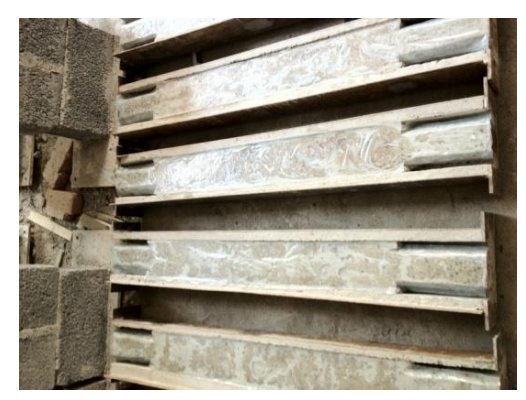

(b) Unbounding process of restraining members after casting concrete

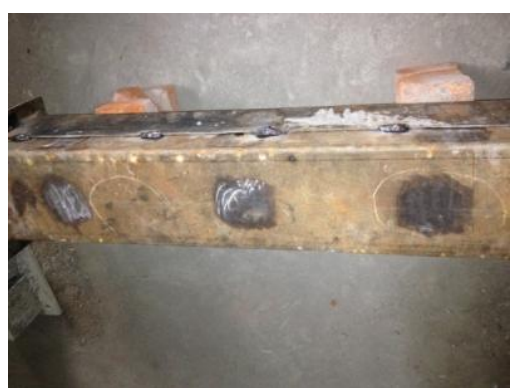

(c) Spot welding on a pair of channel steel components

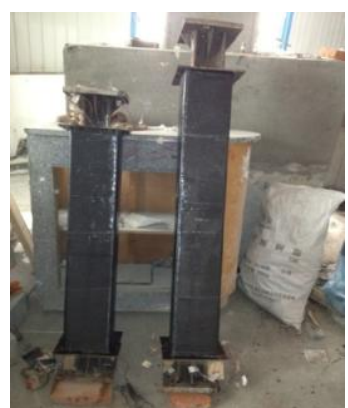

(d) Wrapping fiber cloth

Fig.7. Unbounding process and assembling of BRB specimens

\subsection{Test setup}

The test program was executed in Structure and Mechanics Experiment Center at Harbin Institute of Technology. The specimens were mounted to a Mechanical Testing System (MTS) via of connection seats (see Fig. 8). The loading capacities of MTS are $2500 \mathrm{kN}$ and $2000 \mathrm{kN}$ for compression and tension, respectively, with the loading velocity of $0.18 \mathrm{~mm} / \mathrm{s}$. The applied axial load was controlled by a self-equipped load cell. The corresponding axial deformation of specimen was measured with two displacement transducers (see Fig. 8). 

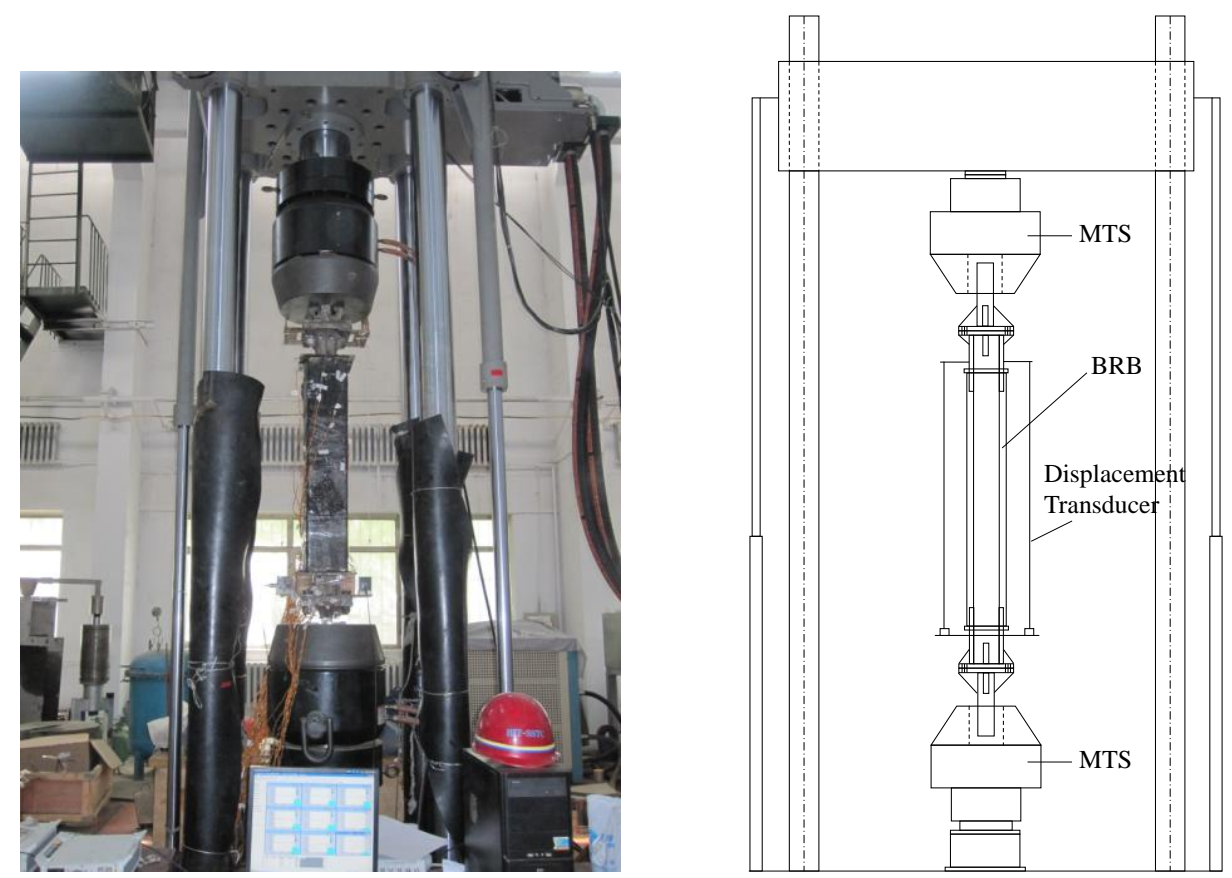

Fig.8. MTS test devices

Two loading protocols were adopted herein, and the increasing displacement cycles are listed in Table 5 and shown in Fig. 9. The first loading protocol includes increasing deformation amplitudes with additional fatigue cyclic loading, while the second one consists of increasing deformation amplitude cyclic loading without maximum amplitude limit. Each loading protocol includes the elastic stage and the after-yielding stage. A force-controlled loading procedure was used in the elastic loading stage, while, in the after-yielding stage, the loading process is controlled with displacement.

Table 5 Loading protocols

\begin{tabular}{|c|c|c|c|c|c|}
\hline \multirow{2}{*}{$\begin{array}{l}\text { Loading } \\
\text { protocols }\end{array}$} & \multirow{2}{*}{$\begin{array}{l}\text { Inter-story } \\
\text { drift ratio }\end{array}$} & \multirow{2}{*}{ Axial strain } & \multicolumn{2}{|c|}{ Axial displacement $(\mathrm{mm})$} & \multirow{2}{*}{ Cycling times } \\
\hline & & & S-specimens & L-specimens & \\
\hline \multirow{6}{*}{ Protocol 1} & $2.5 \%$ & $3.75 \%$ & 2.7 & 3.375 & 3 \\
\hline & $1 \%$ & $7.5 \%$ & 5.4 & 6.75 & 3 \\
\hline & $1.5 \%$ & $1.125 \%$ & 8.1 & 10.125 & 3 \\
\hline & $2 \%$ & $1.5 \%$ & 10.8 & 13.5 & 3 \\
\hline & $2.4 \%$ & $1.8 \%$ & 12.96 & 16.2 & 3 \\
\hline & $2 \%$ & $1.5 \%$ & 10.8 & 13.5 & Keep cycling \\
\hline \multirow[t]{9}{*}{ Protocol 2} & $2.5 \%$ & $1.875 \%$ & 1.35 & 1.6875 & 3 \\
\hline & $5 \%$ & $3.75 \%$ & 2.7 & 3.375 & 3 \\
\hline & $7.5 \%$ & $5.625 \%$ & 4.05 & 5.0625 & 3 \\
\hline & $1 \%$ & $7.5 \%$ & 5.4 & 6.75 & 3 \\
\hline & $1.25 \%$ & $9.375 \%$ & 6.75 & 8.4375 & 3 \\
\hline & $1.5 \%$ & $1.125 \%$ & 8.1 & 10.125 & 3 \\
\hline & $1.75 \%$ & $1.3125 \%$ & 9.45 & 11.8125 & 3 \\
\hline & $2 \%$ & $1.5 \%$ & 10.8 & 13.5 & 3 \\
\hline & $2.4 \%$ & $1.8 \%$ & 12.96 & 16.2 & 3 \\
\hline
\end{tabular}




\begin{tabular}{ccccc}
\hline $2.8 \%$ & $2.1 \%$ & 15.12 & 18.9 & 3 \\
$3.2 \%$ & $2.4 \%$ & 17.28 & 21.6 & 3 \\
$3.6 \%$ & $2.7 \%$ & 19.44 & 24.3 & 3 \\
$4 \%$ & $3.0 \%$ & 21.6 & 27 & 3 \\
\hline
\end{tabular}

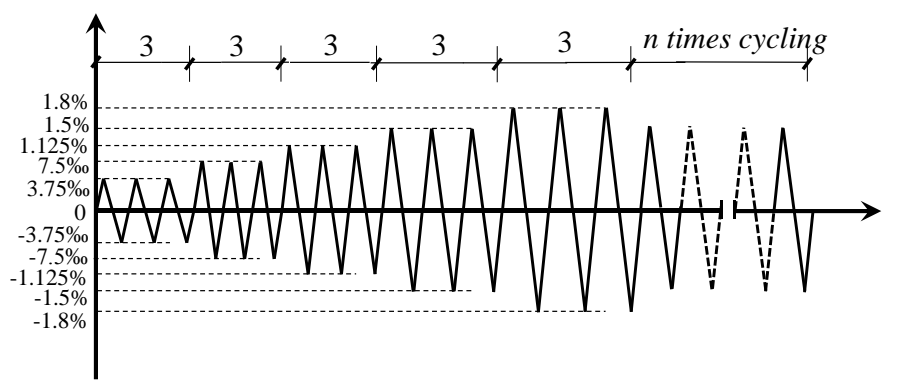

(a) Loading protocol 1

303

\subsection{Test phenomenon}

All eight specimens were found to lose their load-carrying capacity due to rupture of core plates. The tension strength of ABRB specimens dropped drastically when the core plate elements fractured. During loading process, sound can be heard from the tension of fiber and the separation of outer fiber cloth and channel steel component. However, no rupture failure was found at the channel steel and fiber cloth. Therefore, the global buckling failure did not occur in the test program.

Fig. 10 shows the typical rupture phenomenon observed from the core plates of ABRB specimens. It is found that fracture occurred in the middle of the core plates (see Fig. 10(a) (d)) when loading protocol 1 was conducted. Under loading protocol 2, almost all ABRB specimens occurred rupture in the vicinity of the yielding segment ends of core plates except BRB-L-CFRP-2 (see Fig. 10(e) (g)). Besides, cracks were observed at the end of yielding part due to the welding residual stress and stress concentration (see Fig. 

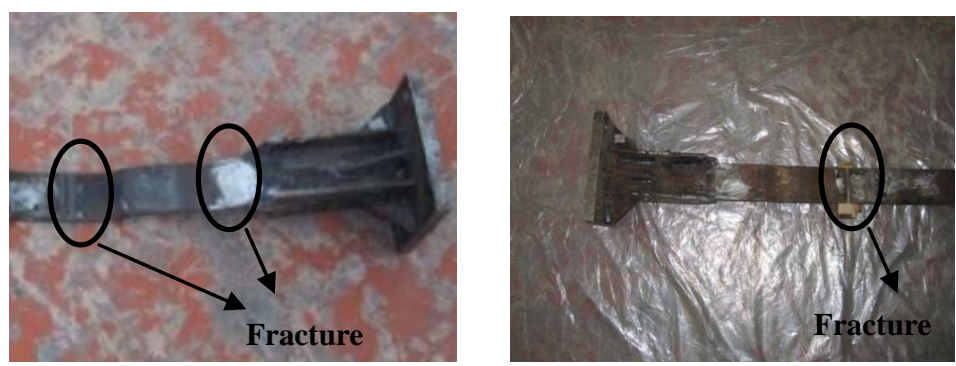

(a) Typical tension fracture of S-Bas-1

(b) Typical tension fracture of S-CFRP-1
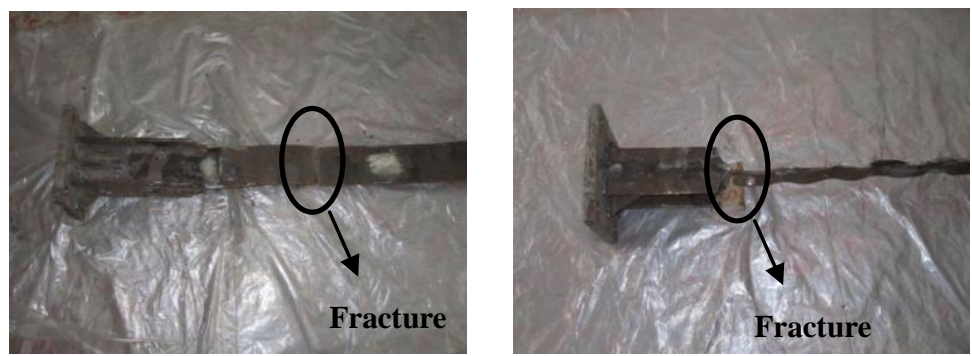

(d) Typical tension fracture of L-CFRP-1

(e) End fracture of S-Bas-2
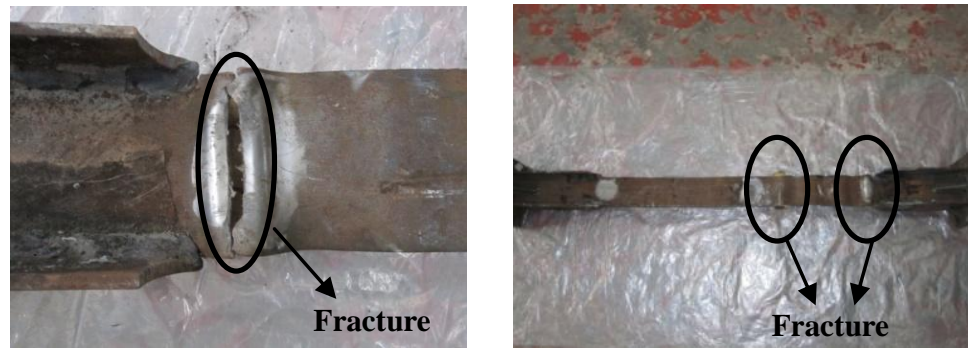

(g) End fracture of L-Bas-2

(h) Typical tension fracture of L-CFRP-2

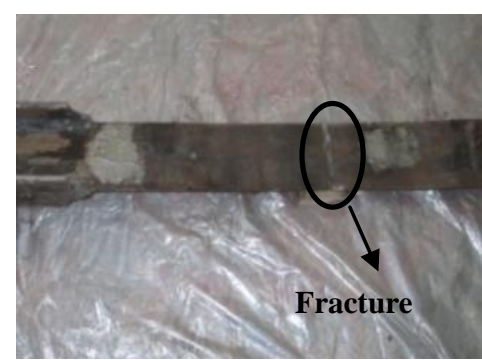

(c) Typical tension fracture of L-Bas-1

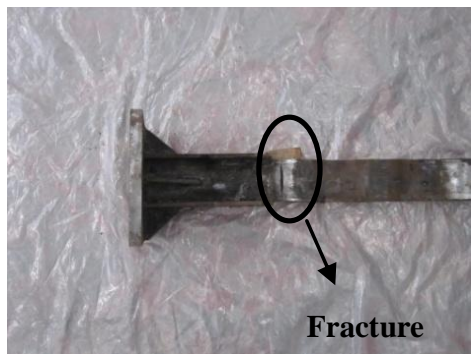

(f) End fracture of S-CFRP-2

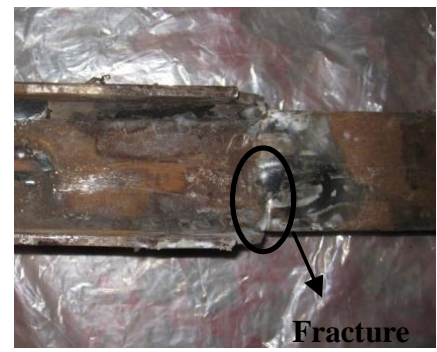

(i) Cracks at the end of BRB specimen

Fig.10. Rupture of load-carrying elements

Fig. 11(a) (h) shows the deformation of core plates that displayed multiple-wave buckling mode. It is clear that the wavelengths are varying with different specimens due to diverse deformation development process caused by different specimen dimensions and different loading protocols. 
334

335

336

337

338

339

340

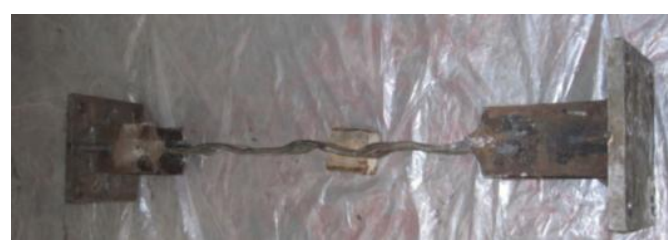

(a) S-Bas-1

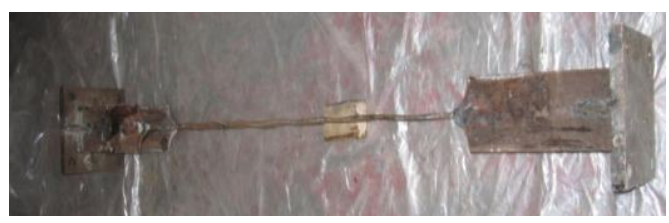

(c) L-Bas-1

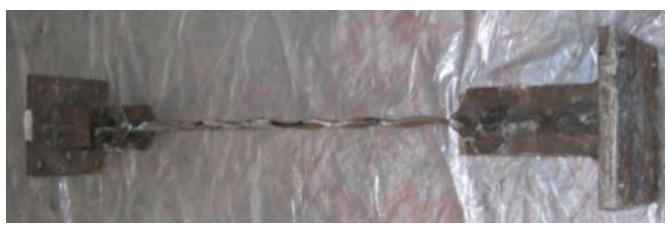

(e) S-Bas-2

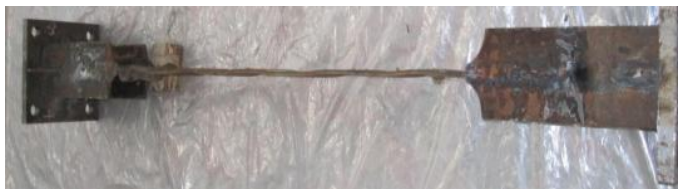

(g) L-Bas-2

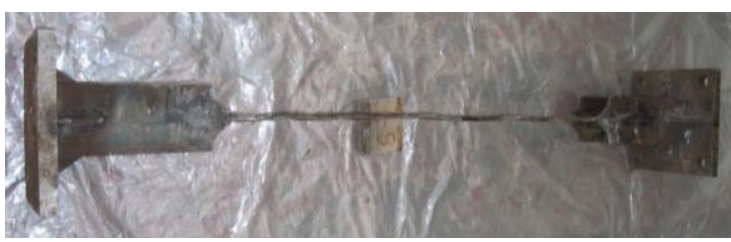

(b) S-CFRP-1

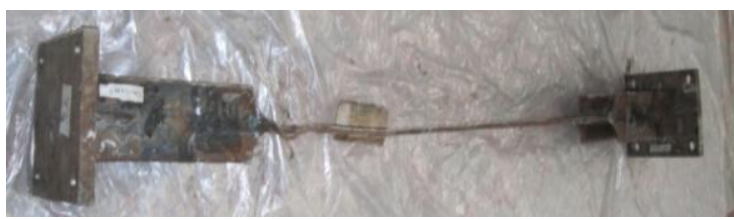

(d) L-CFRP-1

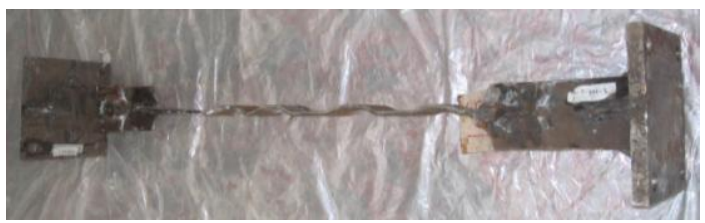

(f) S-CFRP-2

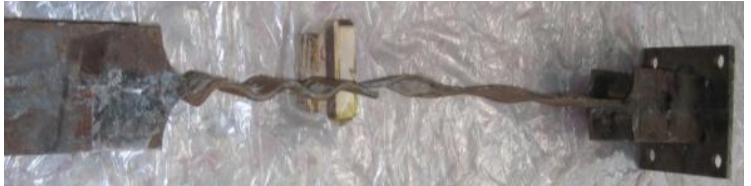

(h) L-CFRP-2

Fig.11. Deformation of core plate elements of BRB specimens cracks, as shown in Fig. 12. Fig. 13 shows that, when the fiber cloth was torn off, the spot welding points and outer channel steel components are generally intact without serious damage. The spot welding points were further cut off to disassemble the BRB specimens. It is found that the infill concrete was almost undamaged without clear cracks, as shown in Fig. 14(a), (b).

After tests, no obvious damage was found on carbon and basalt fiber cloth except for several minor 


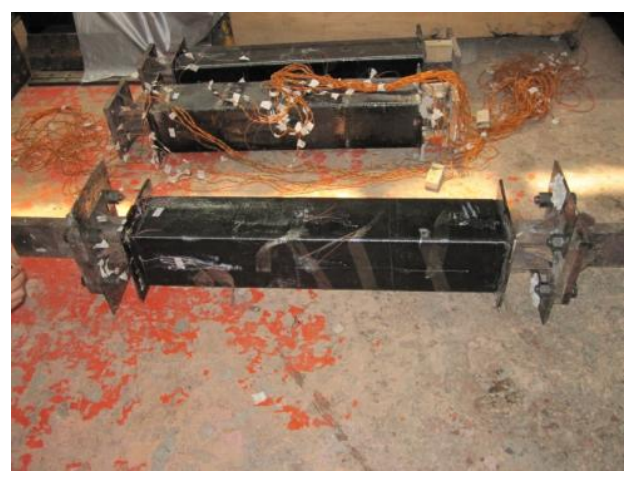

Fig.12. Fiber cloth after test

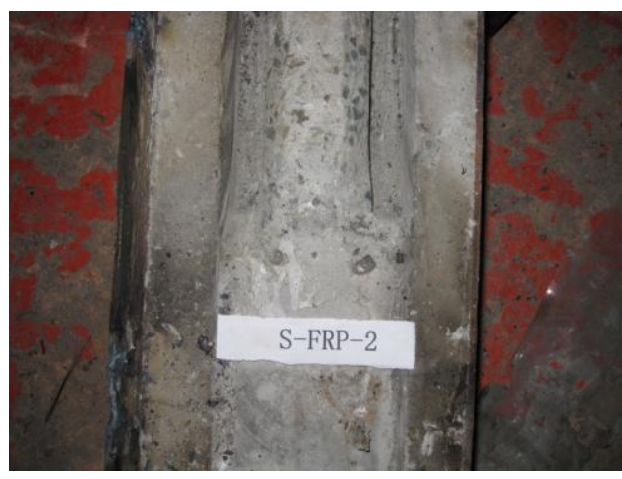

(a) S-CFRP-2

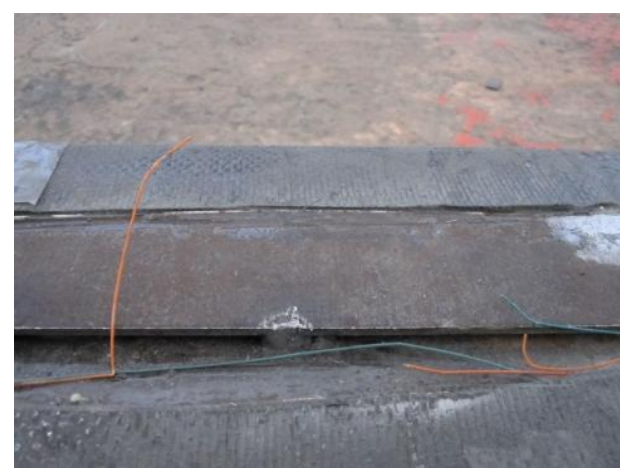

Fig.13. Spot welding points after test

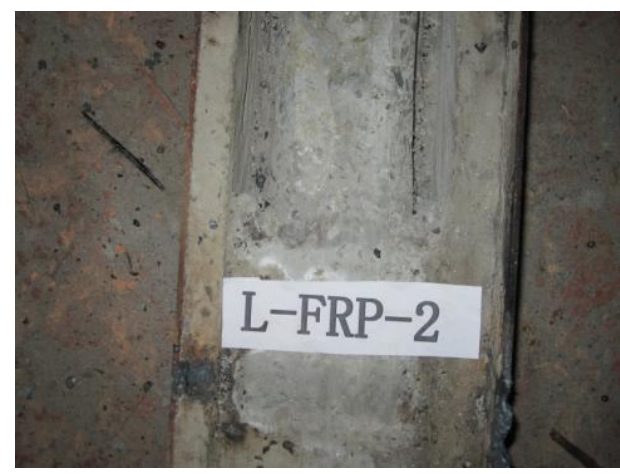

(b) L-CFRP-2

Fig.14. Infilled concrete after test

\subsection{Hysteretic curve}

Fig. 15(a) (l) shows the hysteretic loops of the eight specimens. All specimens show reliable hysteretic behavior before the rupture of the load-carrying element. In the hysteretic curves of specimens S-Bas-1, S-CFRP-1, L-Bas-1, S-CFRP-2, and L-CFRP-2, obvious "local beats" can be observed in the displacement-control compression stage where the stiffness is comparatively small. This observation is due to the multiple-wave buckling of core plates. When multiple-wave buckling appears, core plates of specimens cannot provide sufficient stiffness unless they were straightened. Therefore, the stiffness of core plate stays at a low level during the transformation of the hysteretic curve from the compressive displacement to the tensile displacement. 


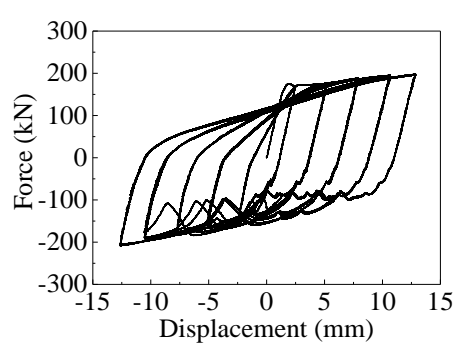

(a) S-Bas-1 (1st stage)

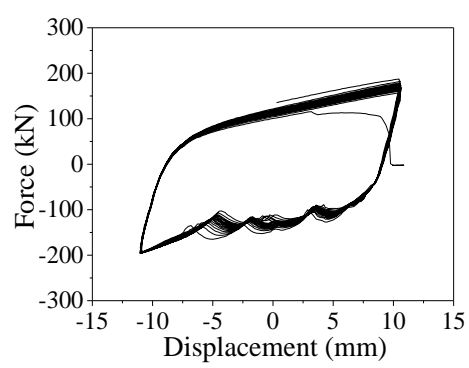

(d) S-CFRP-1 (2nd stage)

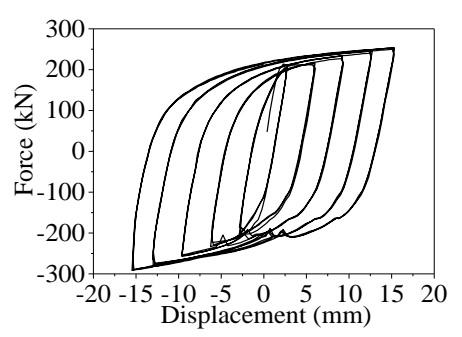

(g) L-CFRP-1 (1st stage)

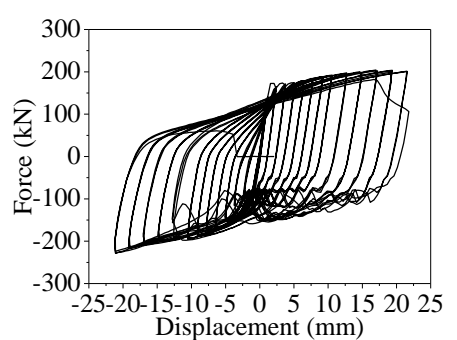

(j) S-CFRP-2

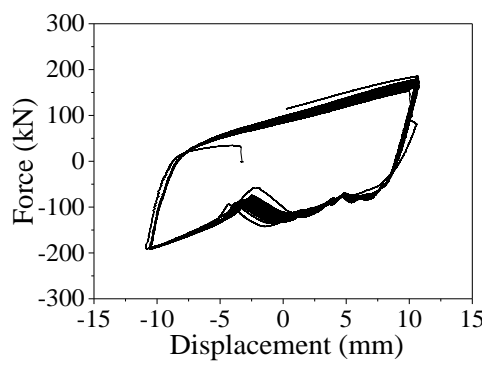

(b) S-Bas-1 (2nd stage)

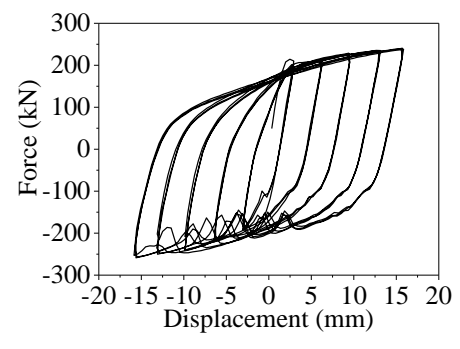

(e) L-Bas-1 (1st stage)

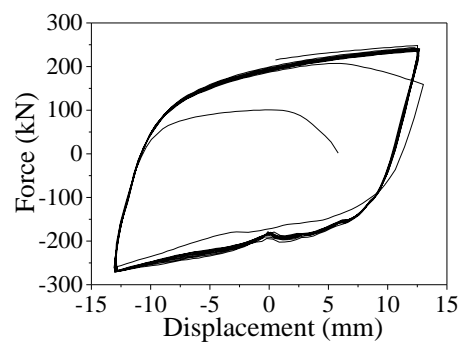

(h) L-CFRP-1 (2nd stage)

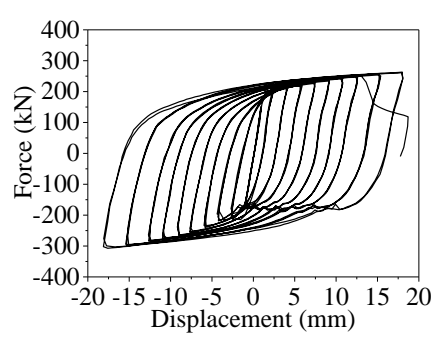

(k) L-Bas-2

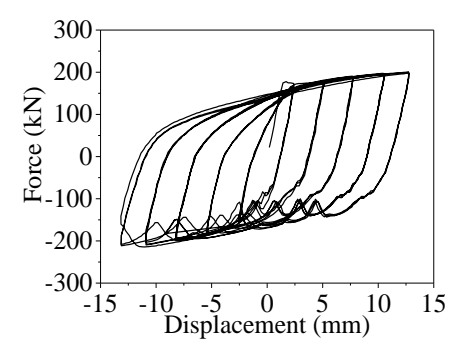

(c) S-CFRP-1 (1st stage)

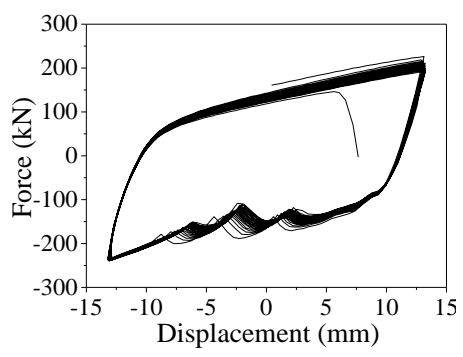

(f) L-Bas-1 (2nd stage)

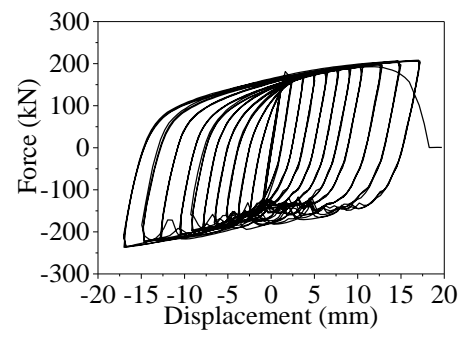

(i) S-Bas-2

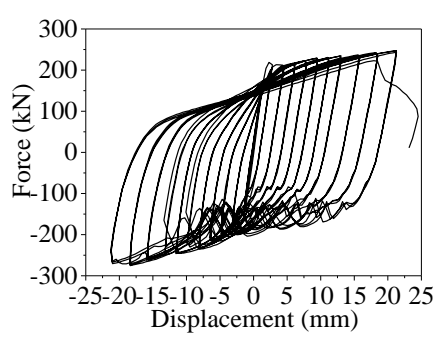

(1) L-CFRP-2

Fig.15. Hysteretic curves of BRB specimens

\subsection{Performance assessment}

The compression strength adjustment factor, $\beta$, and the strain hardening adjustment factor, $\omega$, were calculated for all specimens and shown in Table 6, where $\beta$ and $\omega$ factors are calculated by Eq. (12) and Eq. (13), respectively, 


$$
\begin{gathered}
\omega=\frac{P_{t, \text { max }}}{P_{y}} \\
\beta=\frac{P_{c, \text { max }}}{P_{t, \text { max }}}
\end{gathered}
$$

where $P_{t, \text { max }}$ and $P_{c, \text { max }}$ are the maximum compression and tension strengths of specimens, respectively.

Table 6 shows the calculated $\beta$ and $\omega$ factors, where the calculated $\omega$ factors for all specimens are greater than one and ranges between 1.06 and 1.17, indicating sufficient strain hardening and inelastic deformation development of core plates. The computed $\beta$ factors range between 1.06 and 1.17, which satisfy the upper limit of 1.3 specified in the AISC/SEAOC recommended provisions for buckling-restrained braced frames [25].

Table 6 Calculated $\beta$ and $\omega$ factors for all specimens

\begin{tabular}{ccccccccc}
\hline Specimen & S-Bas-1 & S-CFRP-1 & L-Bas-1 & L-CFRP-1 & S-Bas-2 & S-CFRP-2 & L-Bas-2 & L-CFRP-2 \\
\hline$\omega$ & 1.10 & 1.09 & 1.06 & 1.13 & 1.13 & 1.14 & 1.17 & 1.09 \\
$\beta$ & 1.06 & 1.13 & 1.06 & 1.15 & 1.17 & 1.07 & 1.16 & 1.16 \\
\hline
\end{tabular}

Ductility is one of the parameters which reflect the performance of BRBs, especially a measure to describe the plastic demand on a BRB is the cumulative plastic ductility (CPD), which is a normalized expression defined by:

$$
C P D=\sum_{i} \frac{\left|\Delta_{p i}^{+}-\Delta_{p i}^{-}\right|}{\Delta_{y}}
$$

where $\Delta_{p i}^{+}$and $\Delta_{p i}^{-}$are the maximum values of positive and negative plastic deformations, respectively, during each visit $i$ into the inelastic range; and $\Delta_{y}$ is the yielding deformation of the ABRB specimen. Fig. 16(a), (b) shows the calculated CPD values varying with loading cycle number. It is clear that CPD values increase with the improvement of loading cycling number for both loading protocols. The maximum CPD value and the corresponding loading cycle number are summarized in Table 7. The maximum CPD value of specimen S-Bas-1 reaches 380.17, which is much larger than that of the other specimens under loading protocol 1. The maximum CPD value of specimen S-CFRP-2 goes up to 477.69 , which is the highest value of that of the specimens subjected to loading protocol 2. 


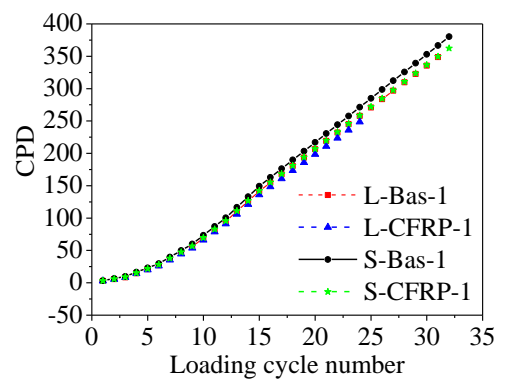

(a) Loading protocol 1

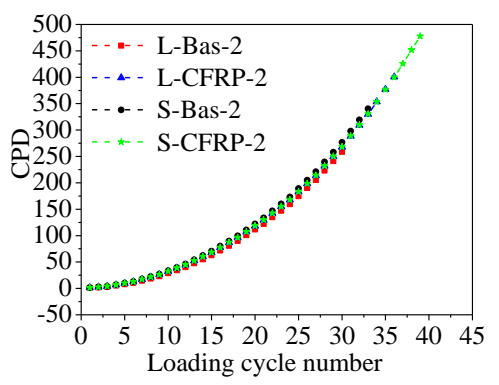

(b) Loading protocol 2

Fig.16. The relationship of CPD and loading cycle number

Table 7 Maximum CPD values for specimens

\begin{tabular}{ccccccccc}
\hline Specimen & S-Bas-1 & S-CFRP-1 & L-Bas-1 & L-CFRP-1 & S-Bas-2 & S-CFRP-2 & L-Bas-2 & L-CFRP-2 \\
\hline Cycling number & 32 & 32 & 31 & 24 & 33 & 39 & 30 & 36 \\
CPD & 380.17 & 362.30 & 348.58 & 248.66 & 340.40 & 477.69 & 258.81 & 400.61 \\
\hline
\end{tabular}

Energy dissipation capacity is an important performance indicator for BRB as an energy consumption component. Four parameters, including secant stiffness $K_{i}$, cumulative energy $E_{h}$, energy dissipation ratio $\psi$, and equivalent viscous damping coefficient $h_{e}$, are used here to measure the energy dissipation capacity of the test specimens. According to the definition in Fig.17, the values of $K_{i}, E_{h}, \psi, h_{e}$ can be calculated by Eq. (15), Eq. (16), Eq. (17) and Eq. (18), respectively.

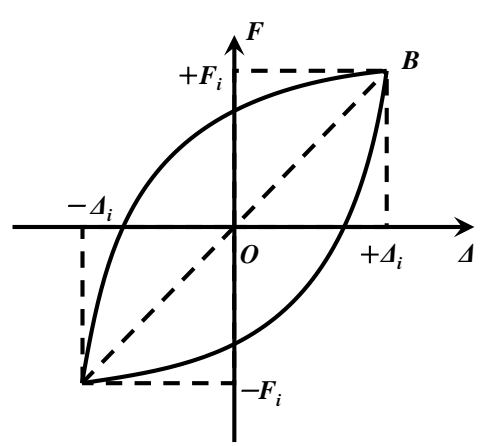

(a) Definition of secant stiffness $K_{i}$

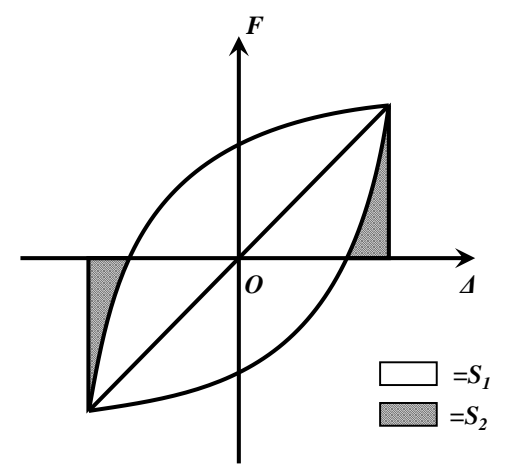

(b) Definition of energy dissipation ratio $\psi$

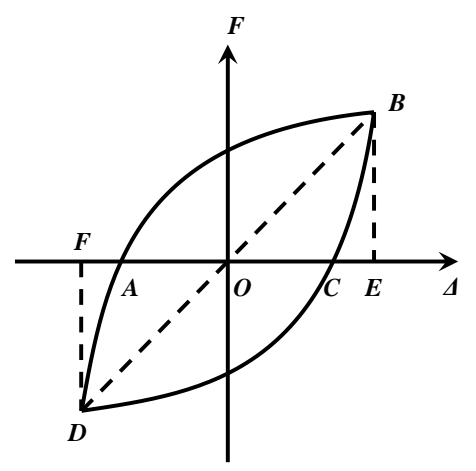

(c) Definition of equivalent viscous damping coefficient $h_{e}$

Fig.17. Definition of energy dissipation indexes and secant stiffness 


$$
K_{i}=\frac{\left|+F_{i}\right|+\left|-F_{i}\right|}{\left|+\Delta_{i}\right|+\left|-\Delta_{i}\right|}
$$

where $K_{i}$ is the secant stiffness in the $i^{\text {th }}$ recorded loop. As shown in Figure 17(a), $F_{i}$ and $\Delta_{i}$ are the maximum load and displacement in the $i^{\text {th }}$ recorded hysteric loop, respectively.

Accumulative energy, $E_{h}$, is the sum of the area enclosed by the force $F$ versus displacement $\Delta$ hysteretic loops, representing the hysteretic energy dissipated by the BRB

$$
E_{h}=\sum_{i} \int F d \Delta
$$

Energy dissipation ratio $\psi$ is defined by dividing dissipated energy by the work of force:

$$
\psi=\frac{S_{1}}{S_{1}+S_{2}}
$$

where $S_{1}$ and $S_{2}$ are the absorbed energy and the discharged energy, respectively (see Fig. 17(b)); and $S_{1+} S_{2}$ are the total work of horizontal force. It is clear that a large value of $\psi$ implies sufficient energy dissipation capacity of the ABRB specimen.

Under cyclic loading condition, strength and stiffness of BRB have degenerated, which is depicted by the equivalent viscous damping coefficient $h_{e}$. The value of $h_{e}$ is represented by the ratio of dissipated energy (area of $\mathrm{ABC}$ and $\mathrm{CDA}$ ) to absorbed energy of equivalent elastic component (area of OBE and ODF) into the same displacement (see Fig. 17(c)), yielding

$$
h_{e}=\frac{1}{2 \pi} \frac{\operatorname{area}(\mathrm{ABC}+\mathrm{CDA})}{\operatorname{area}(\mathrm{OBE}+\mathrm{ODF})}
$$

Fig. 18 and Fig. 19 show the calculated values of $K_{i}, E_{h}, \psi, h_{e}$ varying with the strain of core plates due to loading protocol 1 and 2, respectively. It is clear that the values of $E_{h}, \psi$, and $h_{e}$ increase with the growth of core plate strain, while the values of $K_{i}$ are reduced with the development of strain due to the increasing plastic development of core plate. The minimum values of $K_{i}$, and maximum values of $E_{h}, \psi$ and $h_{e}$ are summarized in Table 8, where the average values of $K_{i}, E_{h}, \psi$ and $h_{e}$ are also calculated for all ABRB specimens. It is seen from the table that the calculated $\psi$ factors are close to 1 , and the computed $h_{e}$ values approach 0.45 , which respectively are the maximum values of energy dissipation ratio and viscous damping coefficient. This result implies that the BRB specimens have good energy dissipation capacity. Moreover, loading protocol 2 leads to larger values of $E_{h}, \psi, h_{e}$ for the ABRB specimens than loading protocol 1. It can also be seen from Table 8 that the initial secant stiffness of S-CFRP-1 is larger than that of S-Bas-1 since the initial defect of S-CFRP-1 is smaller, whereas the calculated values of $\psi, h_{e}$ and $E_{h}$ of S-CFRP-1 

the hysteretic curves of L-CFRP-2 "jump" distinctly under compression, which is accompanied by the

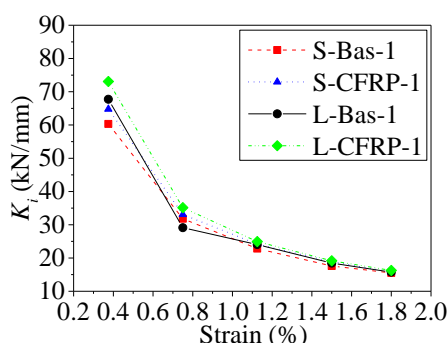

(a) $K_{i}$

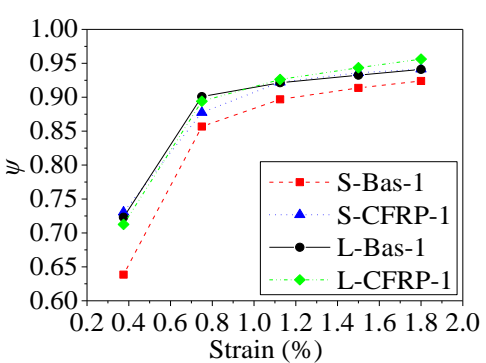

(c) $\psi$

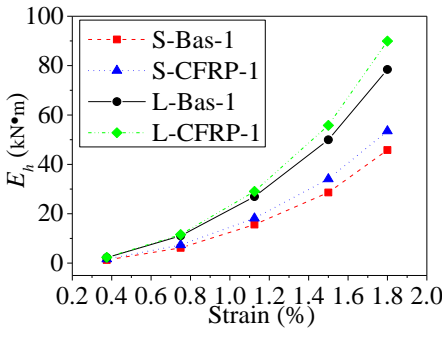

(b) $E_{h}$

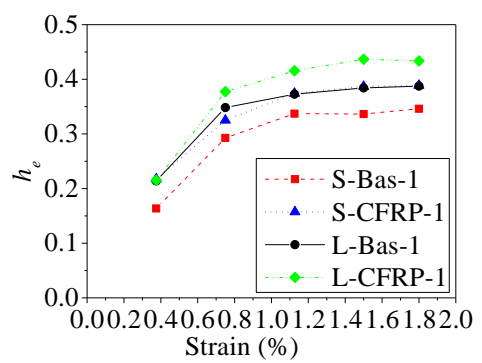

(d) $h_{e}$

Fig.18. Energy dissipation capacity of BRB specimens under loading protocol 1

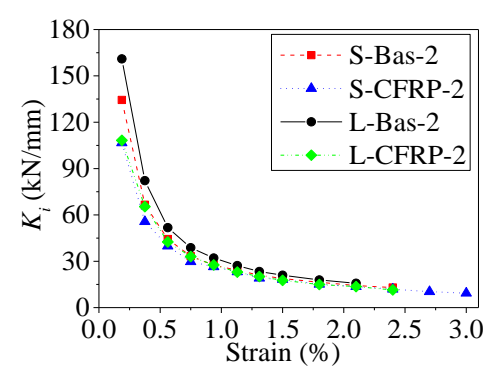

(a) $K_{i}$

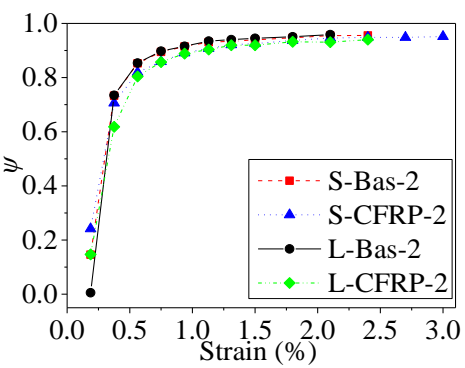

(c) $\psi$

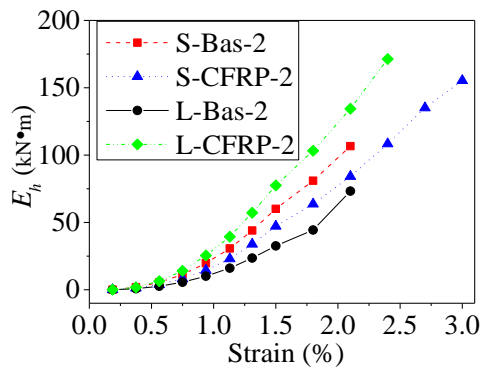

(b) $E_{h}$

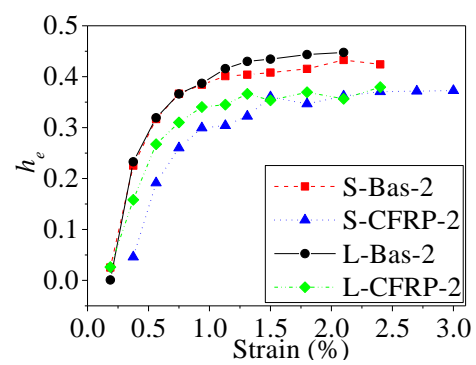

(d) $h_{e}$

Fig.19. Energy dissipation capacity of BRB specimens under loading protocol 2 


\begin{tabular}{|c|c|c|c|c|c|}
\hline $\begin{array}{l}\text { Loading } \\
\text { protocols }\end{array}$ & $\begin{array}{c}\text { BRB } \\
\text { specimens }\end{array}$ & $\begin{array}{c}\text { Minimum } K_{i} \\
(\mathrm{kN} / \mathrm{mm})\end{array}$ & $\begin{array}{l}\text { Maximum } \\
E_{h}(\mathrm{kN} \cdot \mathrm{m})\end{array}$ & Maximum $\psi$ & Maximum $h_{e}$ \\
\hline \multirow{5}{*}{$\begin{array}{l}\text { Loading } \\
\text { protocol } 1\end{array}$} & S-Bas-1 & 15.528 & 45793 & 0.924 & 0.346 \\
\hline & S-CFRP-1 & 15.788 & 53496 & 0.941 & 0.389 \\
\hline & L-Bas-1 & 15.743 & 78458 & 0.941 & 0.388 \\
\hline & L-CFRP-1 & 16.261 & 89950 & 0.956 & 0.434 \\
\hline & $\begin{array}{c}\text { Average } \\
\text { value }\end{array}$ & 15.830 & 66924 & 0.941 & 0.389 \\
\hline \multirow{6}{*}{$\begin{array}{l}\text { Loading } \\
\text { protocol } 2\end{array}$} & S-Bas-2 & 13.003 & 106641 & 0.956 & 0.424 \\
\hline & S-CFRP-2 & 9.335 & 155364 & 0.952 & 0.378 \\
\hline & L-Bas-2 & 15.756 & 73263 & 0.959 & 0.448 \\
\hline & L-CFRP-2 & 11.459 & 171332 & 0.940 & 0.380 \\
\hline & $\begin{array}{c}\text { Average } \\
\text { value }\end{array}$ & 12.388 & 126650 & 0.952 & 0.408 \\
\hline & $\begin{array}{l}\text { Average } \\
\text { value }\end{array}$ & 14.109 & 96787 & 0.947 & 0.399 \\
\hline
\end{tabular}

\section{Finite element analysis}

To provide a better understanding of the compressive load bearing capacity and hysteretic behavior of the presented ABRBs, an analytical study using the finite element computer program ABAQUS was conducted for all ABRB specimens. In the numerical model, the 8-node linear brick with reduced integration (C3D8R) solid element was used to model core plates and infill concrete. The reduced integration (S4R) shell element was used to simulate the nonlinear behaviors of channel steel and fiber cloth. The contact element CONTA174 is applied together with the target element TARGET170 for simulating the 3-dimension surface-to-surface contact phenomenon.

The hard surface-to-surface contact behavior was considered between the core plate and the external restraining members, allowing for separation of the interface in tension and no penetration of that in compression. A frictional coefficient between the contact surfaces of core plate and infill concrete is 0.15 . The distribution and amplitudes of normal contact forces between the contact surfaces is not significantly affected by the friction forces. The confined channel steel was tied with both the infill concrete and the wrapped fiber cloth to avoid relative movement between each other. 
together. The initial imperfection deformation of the core plate is located in the middle as a sine deformation mode, which was adopted by scaling the first buckling mode. A large enough air gap size between the core plate and the concrete is set for developing the geometrically nonlinear phenomenon, but does not cause initial convergence problems due to contact.

Fig. 20 shows the simulated Mises stress contour plot. The significant plasticity appears in the core plate. The plastic strain distribution is uniform along the full length of the yielding zone of the core plate. The biggest stress occurs in the yielding zone of the core (red in Fig. 20(a)), which is decreased at the end of the yielding zone of the core plate due to the enlarged cross section. Significant contact forces appear between the core plate and the restraining members due to local buckling and multi-wave buckling, which lead to the friction stresses around both axes. The biggest stress, which is caused by the global flexural deformation and contact forces between the core plate and the restraining members, occurs in the middle corner region of the confined concrete infilled channel steel and fiber cloth (red in Fig. 20(b), (c)).

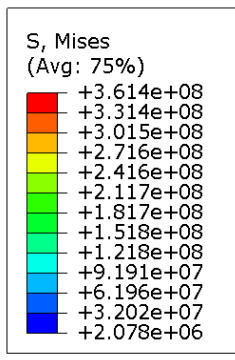

(a) Core plate
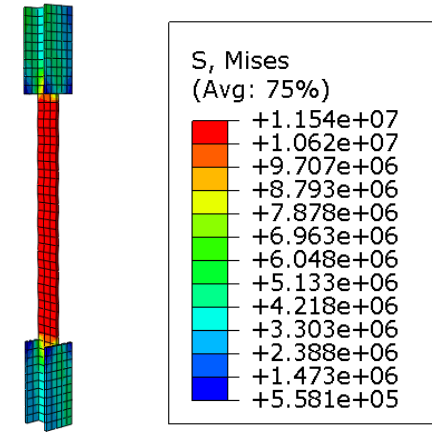

(b) Concrete
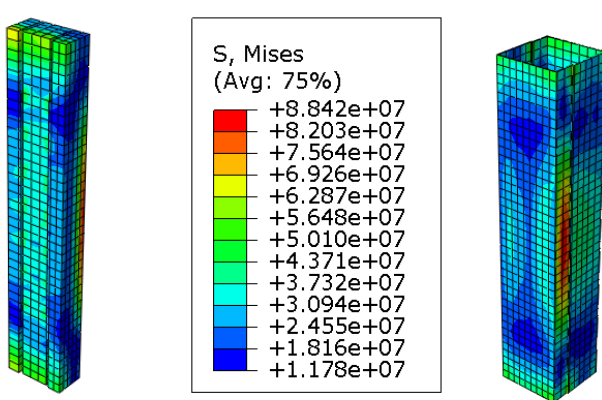

(c) Fiber cloth

Fig.20. The maximum compression stress $\left(\mathrm{N} / \mathrm{m}^{2}\right)$ contours of BRB

The simulated maximum compression stress contours of L-Bas-1, L-CFRP-1, S-BAS-2 and L-BAS-2 are compared with the experimental observations and shown in Fig. 21 24. The experiment results show that core plates cracked at the middle or the end of yielding segments. This observation can also be reflected from the finite element analysis results where the maximum stress is located in the same position of the core plate. The consistence between experiment and finite element analysis shows the effectivity of the finite element model at representing the failure mode of the ABRB specimens. Both test and analysis display obvious multi-wave buckling that occurred on the core plates. Besides, the simulated deformation mode of core plate is consistent with that observed from experiments. 


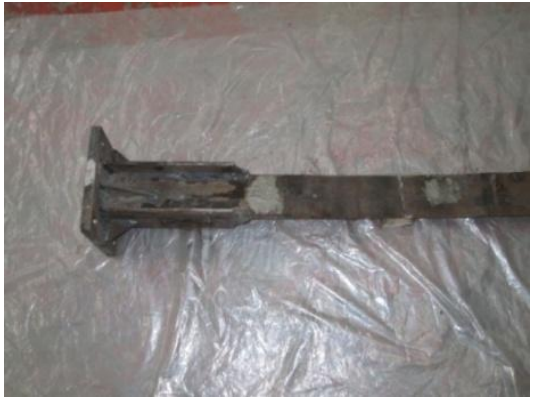

(a) Experiment phenomenon
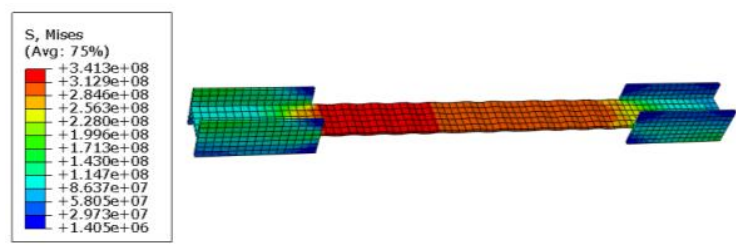

(b) Finite element analysis stress $\left(\mathrm{N} / \mathrm{m}^{2}\right)$ contours

Fig.21. Comparison of experiment phenomenon and finite element analysis stress contours of BRB-L-Bas-1

497

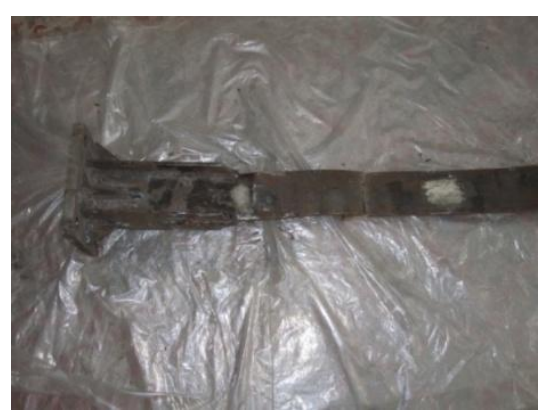

(a) Experiment phenomenon
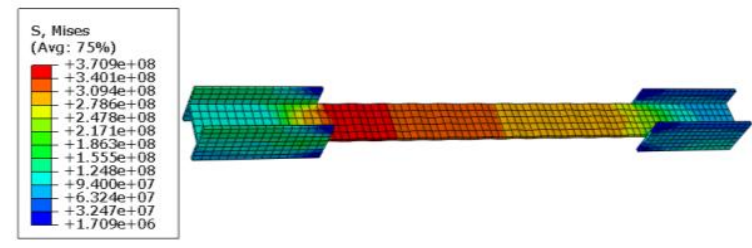

(b) Finite element analysis stress $\left(\mathrm{N} / \mathrm{m}^{2}\right)$ contours

Fig.22. Comparison of experiment phenomenon and finite element analysis stress contours of BRB-L-CFRP-1

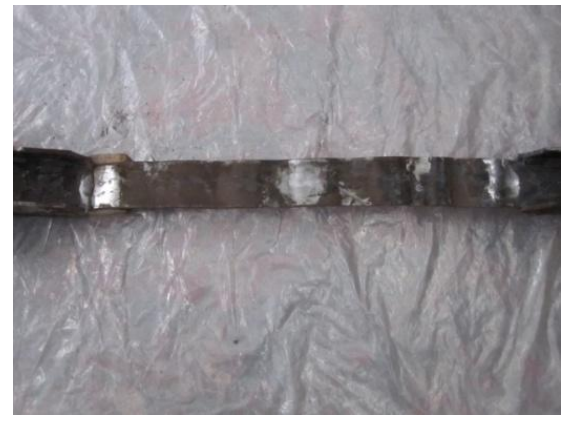

(a) Experiment phenomenon

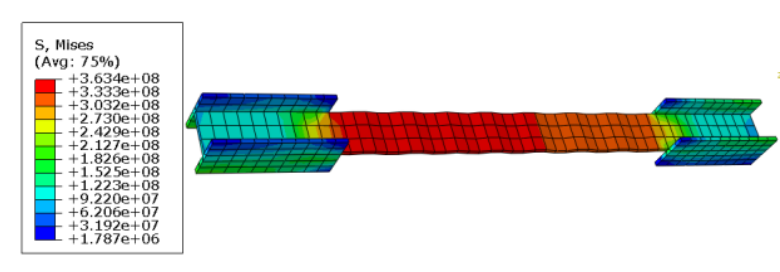

(b) Finite element analysis stress $\left(\mathrm{N} / \mathrm{m}^{2}\right)$ contours

Fig.23. Comparison of experiment phenomenon and finite element analysis stress contours of BRB-S-BAS-2 


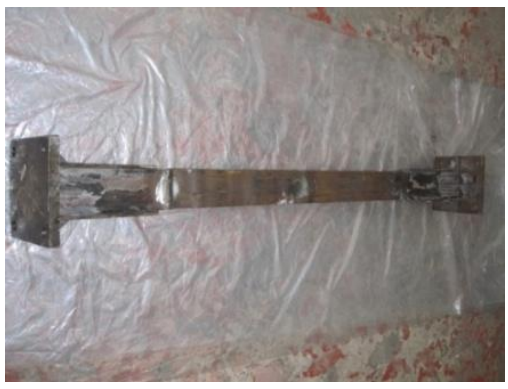

(a) Experiment phenomenon

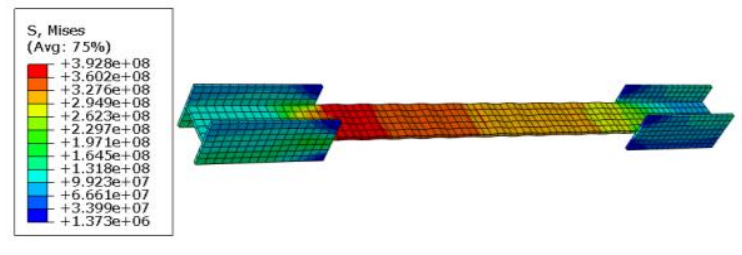

(b) Finite element analysis stress $\left(\mathrm{N} / \mathrm{m}^{2}\right)$ contours

Fig.24. Comparison of experiment phenomenon and finite element analysis stress contours of BRB-L-BAS-2

500

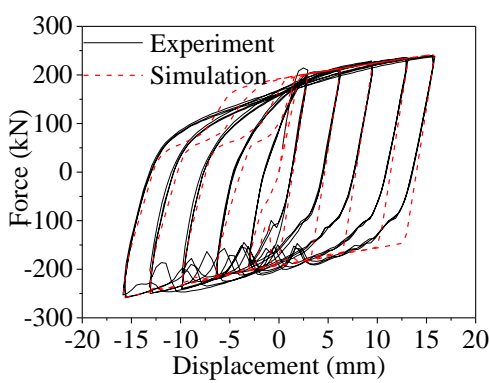

(a) BRB-L-Bas-1

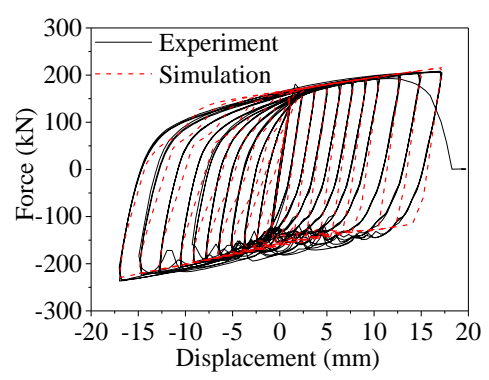

(c) BRB-S-BAS-2

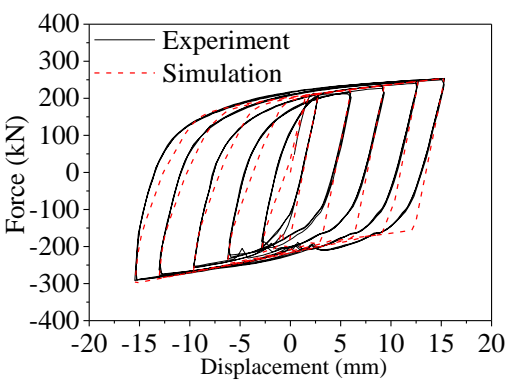

(b) BRB-L-CFRP-1

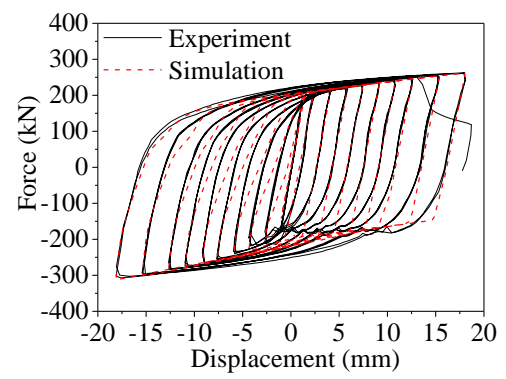

(d) BRB-L-BAS-2

Fig.25. Comparison of experiment and simulation hysteretic curves of BRB specimens

From the hysteretic model and framework curve of axial deformation with axial force in the finite element simulations and tests, the resilience model (normalized simple hysteretic model) is ensured as the backbone curves. The backbone curves shown in Fig. 26 indicate that a bilinear model was proposed to approximate the macroscopic behavior of BRB specimens. The compression stiffness and strength is larger than that of tension, the major factor that influenced the imbalanced load is that the significant lateral 

compression.

513

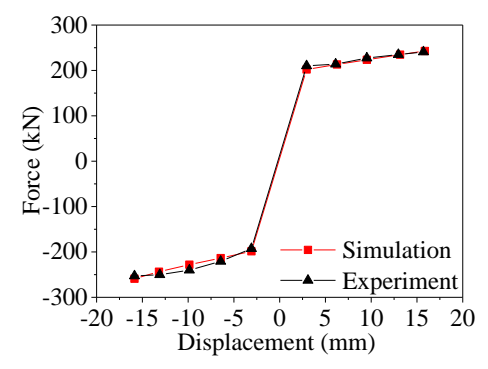

(a) BRB-L-Bas-1

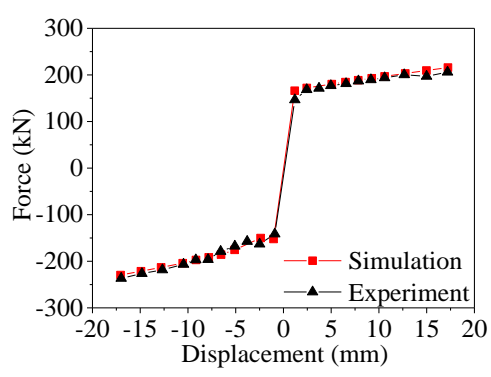

(c) BRB-S-BAS-2

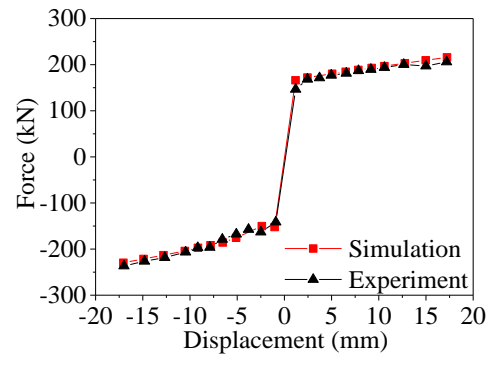

(b) BRB-L-CFRP-1

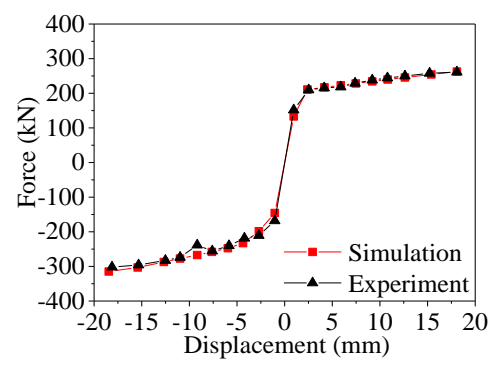

(d) BRB-L-BAS-2

Fig.26. Comparison of experiment and simulation backbone curves of BRB specimens

The simulated hysteretic curves and backbone curves are almost identical with that from experiments.

As shown in Fig. 27, 28, the equivalent viscous damping coefficient $h_{e}$ and secant stiffness $K_{i}$ calculated from simulation show good agreement with that from the experimental results. The $h_{e}$ increase and $K_{i}$ decrease with the growth of strain increment. The increasing plastic development resulted smaller $K_{i}$, fuller hysteretic curves and bigger $h_{e}$. The analytical results show that the adopted FE models of the ABRBs can simulate their performance well and can be used for further parametric study. 


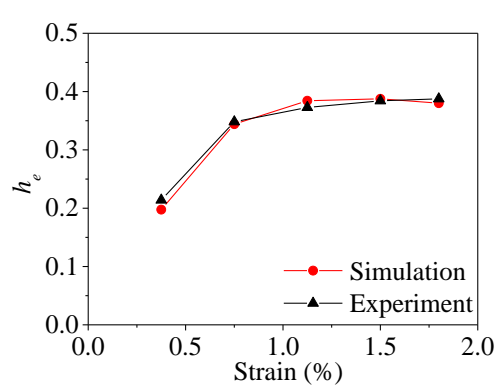

(a) BRB-L-Bas-1

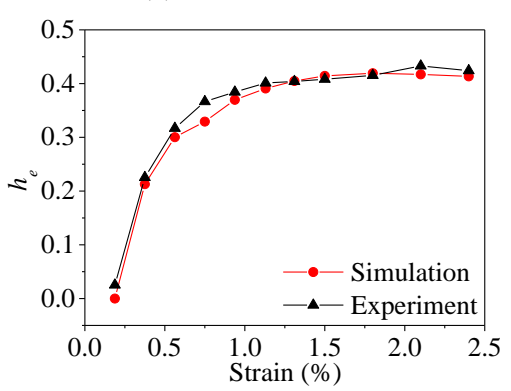

(c) BRB-S-BAS-2

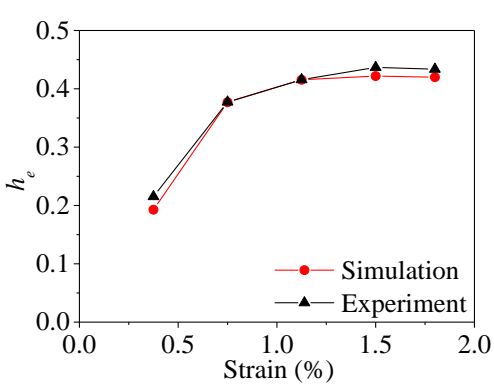

(b) BRB-L-CFRP-1

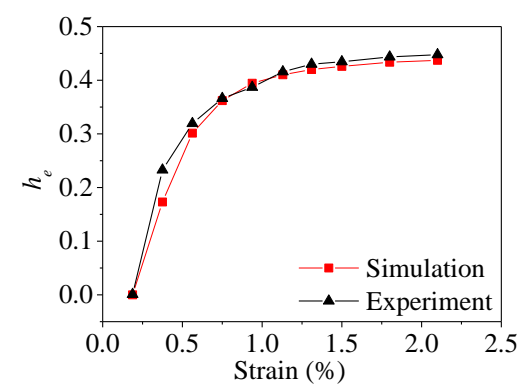

(d) BRB-L-BAS-2

Fig.27. Comparison of experiment and simulation equivalent viscous damping coefficient of BRB specimens

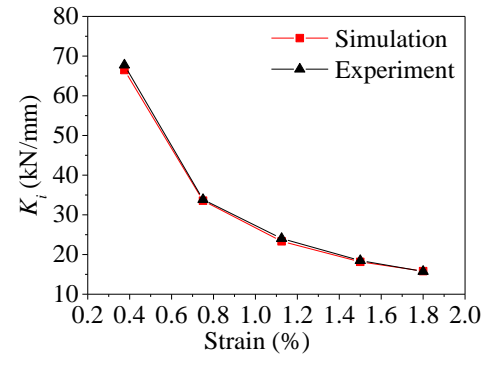

(a) BRB-L-Bas-1

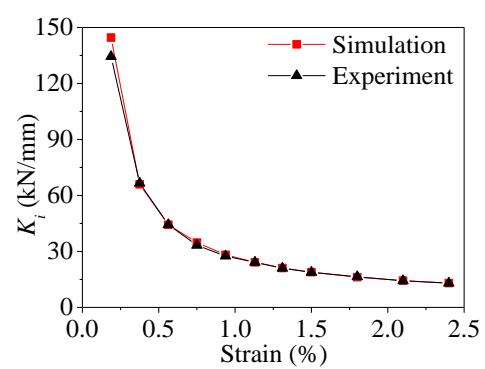

(c) BRB-S-BAS-2

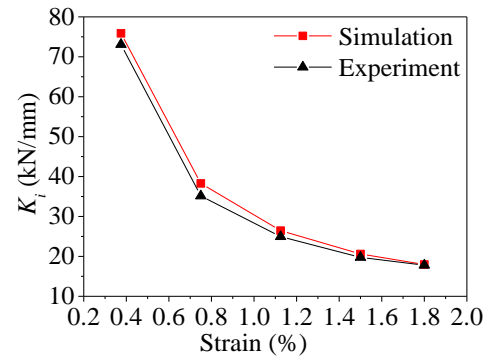

(b) BRB-L-CFRP-1

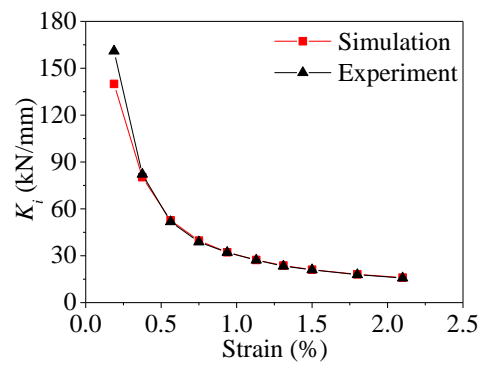

(d) BRB-L-BAS-2

Fig.28. Comparison of experiment and simulation equivalent secant stiffness of BRB specimens

\section{Conclusions}

A new assembled buckling-restrained brace (ABRB) wrapped with carbon or basalt fiber cloth is proposed. To examine the performance of the newly presented ABRB, eight specimens were tested under cyclic loading. The ABRB specimens show good performance at the hysteretic behavior, stiffness, strength, ductility and energy absorption capacity. A pair of restraining members of the ABRB can be connected and restrained with carbon or basalt fiber cloth effectively and efficiently. Therefore, the assembled restraining 
members can resist the lateral thrust exerted by core plate as a whole, and the ABRB can prevent the global buckling with enough stiffness. The test results imply that it is a good alternative assemble method for ABRBs by wrapping fiber cloth around the outer restraining members.

All eight specimen occurred fracture at the core plates. The hysteretic curves of specimen "jump" in the compression stage because multi-wave buckling has appeared at core plates. The tension strength adjustment factors of the specimen range between 1.06 and 1.17. The compression strength adjustment factors are computed between 1.06 and 1.17 which satisfies the upper limit requirement of 1.3 specified in the AISC/SEAOC code. The secant stiffness of the specimen declines with the increase of plastic deformation. The BRB specimens fractured in the middle of the core plate elements under protocol 1; the rupture of the specimens occurred in the junctions of yielding segments and non-yielding segments under protocol 2. The new ABRBs exhibit excellent energy dissipation capacity since the maximum values of energy dissipation ratios are almost greater than 0.92 , and the equivalent viscous damping coefficients is nearly beyond 0.38 .

The experimental results are validated with the finite element analysis, the simulated deformation mode and stress distribution of BRB specimens are consistent with that observed from experiments. The simulated hysteretic curves and backbone curves are almost identical with that of experiments, the viscous damping coefficients and secant stiffness of simulation show good agreement with the experimental results.

\section{Acknowledgements}

The support of National Science Foundation of China through projects (Grant No. 51008090, 51378162, 51408155, 51678209), National Science and Technology Support Project of Ministry of science and technology (2013BAJ08B01), Scientific Research Foundation of Returned Overseas Scholars of the Ministry of Education of China (Grant No. 2014), Scientific Research Foundation of Returned Overseas Scholars of Heilongjiang Province of China (Grant No. 40000045-6-15455). All the opinions, findings, and conclusions or recommendations expressed in this material are those of the authors and do not necessarily reflect those of the Foundations.

\section{References}

[1] Xie, Q. State of the art of buckling-restrained braces in Asia, Journal of Constructional Steel Research, 61(6) (2005) 
[2] Judd J. P., Charney F.A. Seismic collapse prevention system for steel-frame buildings, Journal of Constructional Steel Research, 118(2016) 60-75.

[3] Deylami A., Mahdavipour M.A. Probabilistic seismic demand assessment of residual drift for Buckling-Restrained Braced Frames as a dual system, Structural Safety, 58(2016) 31-39.

[4] Watanabe A., Hitomi Y., Saeki, E. Wada A., Fujimoto M. Properties of braces encased in buckling-restrained concrete and steel tube, Proceeding of ninth world Conference on Earthquake Engineering (Volume IV), Tokyo-Kyoto, Japan, 1998, pp. 719-724.

[5] Chen C.C., Chen S.Y., Liaw J.J. Application of low yield strength steel on controlled plasticization ductile concentrically braced frames, Canadian Journal of Civil Engineering, 28(5) (2001) 823-836.

[6] Wang J.J., Shi Y.J., Wang Y.Q. Constitutive model of low-yield point steel and its application in numerical simulation of buckling-restrained braces, Journal of Materials in Civil Engineering, ASCE 28(3) (2016) 04015142-1-13.

[7] Lai J.W., Tsai K.C. Research and application of buckling restrained braces in Taiwan, ANGER Annual Meeting, Hawaii, USA, 2004.

[8] Black C.J., Makris N., Aiken I.D. Component testing seismic evaluation and characterization of buckling- restrained

[9] Jia M.M., Lu D.G., Zhang S.M., Sun L. Performance testing and cyclic behavior of buckling-restrained braces with H cross section unrestrained segments, Advances in Structural Engineering, 17(5) (2014) 677-692.

[10] Jia M.M., Guo L.H., Lu D.G. Performance testing and comparison of buckling-restrained braces with $\mathrm{H}$ and criss cross section unrestrained segments, International Journal of Steel Structures, 14(4) (2014) 745-753.

[11] Genna F., Gelfi P. Analysis of the lateral thrust in bolted steel buckling-restrained braces. I: experimental and numerical results, Journal of Structural Engineering, ASCE 138(10) (2012) 1231-1243.

[12] Wu A.C., Lin P.C., Tsai K.C. High-mode buckling responses of buckling-restrained brace core plates, Earthquake Engineering \& Structural Dynamics, 43(3) (2014) 375-393.

[13] Iwata M., Murai M. Buckling-restrained brace using steel mortar planks: performance evaluation as a hysteretic damper, Earthquake Engineering \& Structural Dynamics, 35(14) (2006) 1807-1826.

[14] Chou C.C., Chen S.Y. Subassemblage tests and finite element analyses of sandwiched buckling-restrained braces, Engineering Structures, 32(8) (2010) 2108-2121.

[15] Eryasar M.E., Topkaya C. An experimental study on steel-encased buckling-restrained brace hysteretic dampers, 
[16] Hoveidae N., Rafezy B. Overall buckling behavior of all-steel buckling restrained braces, Journal of Constructional Steel Research, 79 (2012) 151-158.

[17] Usami T., Wang C.L., Funayama J. Developing high-performance aluminum alloy buckling-restrained braces based on series of low-cycle fatigue test, Earthquake Engineering \& Structural Dynamics, 41(4) (2012) 643-661.

[18] Wang C.L., Usami T., Funayama J., Evaluating the influence of stoppers on the low-cycle fatigue properties of high-performance buckling-restrained braces, Engineering Structures, 41 (2012) 167-176.

[19] Wang C.L., Usami T., Funayama J., Imase F. Low-cycle fatigue testing of extruded aluminum alloy buckling-restrained braces, Engineering Structures, 46 (2013) 294-301.

[20] Dusicka P., Wiley B. Concept of buckling restraint of steel braces with fiber reinforced polymers, Proceedings of the 2008 Structures Congress, Vancouver, British Columbia, Canada, 2008.

[21] Dusicka P., Tinker J. Global restraint in ultra-lightweight buckling-restrained braces, Journal of Composites for Construction, ASCE 17(1) (2013) 139-150.

[22] Deng K.L., Pan P. Study of GFRP steel buckling restraint braces, Journal of Composites for Construction, ASCE 19(6) (2015) 04015009-1-8.

[23] Industry standard of the People's Republic of China. Technical specification for steel structure of tall buildings (JGJ 99-2015). Ministry of Housing and Urban-Rural Development of the People's Republic of China, China Architecture \& Building Press, Beijing, 2015.

[24] Building Seismic Safety Council. NEHRP Recommended Provisions for Seismic Regulations for New Buildings and Other Structures (FEMA450). National Institute of Building Sciences, Washington, D.C, 2004.

[25] Standard of Engineering Construction Standardization Association of China. Technical specification for structures with concrete-filled rectangular steel tube members (CECS 159:2004). Engineering Construction Standardization Association of China, Beijing, 2004.

[26] The national standard of the People's Republic of China. Code for design of steel structures (GB 50017-2003). Ministry of construction of the People's Republic of China, Beijing, 2003.

[27] The national standard of the People's Republic of China. Code for design of concrete structures (GB 50010 - 2010). Ministry of Housing and Urban-Rural Development of the People's Republic of China, China Architecture \& Building Press, Beijing, 2015.

[28] American Institute of Steel Construction / Structural Engineers Association of Northern. AISC/SEAOC Recommended Provisions for Buckling-Restrained Braced Frames, Seismology and Structural Standards Committee, California, USA, 2001. 